\title{
Can linear transportation infrastructure verges constitute a habitat and/or a corridor for vertebrates in temperate ecosystems? A systematic review
}

Dakis-Yaoba Ouédraogo 1* (1), Anne Villemey', Sylvie Vanpeene ${ }^{2}$, Aurélie Coulon ${ }^{3,4}$, Vital Azambourg1, Marine Hulard ${ }^{1}$, Eric Guinard ${ }^{5}$, Yves Bertheau ${ }^{3,6}$, Frédérique Flamerie De Lachapelle ${ }^{7}$, Vanessa Rauel ${ }^{5}$, Eric Le Mitouard ${ }^{5}$, Arzhvaël Jeusset ${ }^{1}$, Marianne Vargac ${ }^{1}$, Isabelle Witté ${ }^{1}$, Hervé Jactel ${ }^{8}$, Julien Touroult ${ }^{1}$, Yorick Reyjol ${ }^{1}$ and Romain Sordello ${ }^{1 *}$

\begin{abstract}
Background: Linear transportation infrastructures (roads, railways, oil and gas pipelines, powerlines and waterways) generate well documented fragmenting effects on species habitats. However, the potential of verges of linear transportation infrastructures (road and railway embankments, strips of grass under power lines or above buried pipelines, or waterway banks) as habitat or corridor for biodiversity, remains controversial. In a context of constant loss of natural habitats, the opportunities of anthropogenic areas for compensating the loss of biodiversity they generated have to be considered. This paper is the first synthesis of evidence addressing this topic for vertebrates (mammals, birds, amphibians and reptiles) in temperate ecosystems.
\end{abstract}

Methods: We conducted a systematic literature survey using two online publication databases, three search engines, specialist websites, and by sending a call for literature to subject experts. We successively screened the articles for relevance on titles, abstracts and full texts using criteria detailed in an a priori protocol. We then used six specific questions to categorize the retained studies and to critically appraise them. These questions encompassed the potential of verges as habitats and corridors for vertebrates, and the effects of landscape and management on these potentialities. We critically appraised all studies to assess their risk of bias and created a database of the studies with low and medium risk of bias. We synthesized results for each specific question in narrative syntheses. Finally, studies that met meta-analysis requirements were used for quantitative syntheses.

Results: Our initial searches identified 83,565 documents. After critical appraisal, we retained 119 documents that reported 128 studies. Most studies were conducted in Europe (49\%) and in the United States of America (22\%), and were about mammals (61\%) and birds (20\%). Results from the narrative synthesis and meta-analyses converged and revealed that the potential of linear transportation infrastructures verges to constitute a habitat for vertebrate species varies according to the infrastructure and the biological group considered. Especially, highway verges may be a refuge for small mammals but seems detrimental to birds. The potential also varied depending on the landscape considered, with urbanisation being related to lower biodiversity hosted by verges. We found a wide variety of verge

\footnotetext{
*Correspondence: dakis.ouedraogo@gmail.com; romain.sordello@mnhn.fr

1 UMS PatriNat, OFB - MNHN - CNRS, 75005 Paris, France

Full list of author information is available at the end of the article
}

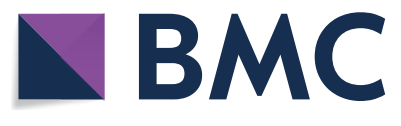

(c) The Author(s) 2020. This article is licensed under a Creative Commons Attribution 4.0 International License, which permits use, sharing, adaptation, distribution and reproduction in any medium or format, as long as you give appropriate credit to the original author(s) and the source, provide a link to the Creative Commons licence, and indicate if changes were made. The images or other third party material in this article are included in the article's Creative Commons licence, unless indicated otherwise in a credit line to the material. If material is not included in the article's Creative Commons licence and your intended use is not permitted by statutory regulation or exceeds the permitted use, you will need to obtain permission directly from the copyright holder. To view a copy of this licence, visit http://creativeco mmons.org/licenses/by/4.0/. The Creative Commons Public Domain Dedication waiver (http://creativecommons.org/publicdomain/ zero/1.0/) applies to the data made available in this article, unless otherwise stated in a credit line to the data. 
management practices with few studies on each practice, which prevented us from drawing general conclusions. Likewise, we found too few studies assessing the corridor potential of verges to be able to fully conclude although this potential seems to exist. We did not find any study assessing the effect of landscape context or management on the role of corridor of verges.

Conclusions: We identified a major knowledge gap regarding the potential of linear transportation infrastructure verges as corridors for vertebrates, and when they exist studies rarely directly measured movements on verges. We thus encourage more research on this topic and the development of protocols that enable direct measures of vertebrate movements. The effect of management practices on the role of habitat of verges also deserves further investigations, and research efforts should be coordinated to focus on one specific practice (e.g. vegetation management).

Keywords: Amphibians, Bats, Biodiversity, Birds, Green infrastructure, Mammals, Refuge, Reptiles, Right of way, Roadside

\section{Background}

For the last decades, human activities have resulted in a global continuous loss of biodiversity [1], and transportation was identified as one of the ten major threats faced by threatened or near-threatened species [2]. Linear transportation infrastructures (LTIs) have led to habitat loss and degradation, fragmentation and barrier effects, light and noise disturbance, chemical pollution and direct mortality (e.g. road kill, electrocution) [3-8]. In particular, the splitting of natural habitats and ecosystems into smaller and more isolated patches (i.e. fragmentation) and the associated loss of habitat have negative effects on biodiversity [9]. For instance, LTIs have induced a decrease in wildlife species abundance at local and large scales [10]. And, through barrier effects LTIs can restrict wildlife movements, disrupt gene flow and metapopulation dynamics, and lead to the genetic isolation of populations over several generations (e.g. [11]).

Considered transversally, LTIs thus generate a demonstrated negative fragmenting effect on biodiversity, but considered longitudinally, LTIs have the potential to constitute a habitat and/or movement corridors for biodiversity by their semi-natural verges [12]. Indeed, inside the LTI boundaries there is generally a transportation lane (road, railway, pipeline, powerline, river or canal) and verges which are most often covered with vegetation (road and railway embankments, strips of grass under power lines or above buried pipelines, or waterway banks, etc.). Studies assessing the potential of LTI verges as habitat and/or corridor for wildlife species have provided contrasted results. For instance, it has been shown that revegetated highway verges could serve as ecological corridors for native rodents and fragmentation-tolerant bird species in California, United States of America [13]. At the opposite, a meta-analysis revealed that densities of mammals and birds, but not raptors, declined in infrastructures verges [10]. The potential of LTI verges as habitat and/or corridor for wildlife species may also vary with verge management practices, as one practice (e.g. mowing) can be beneficial for some species (e.g. disturbance-tolerant species) but not for others (e.g. woody species) $[14,15]$.

Knowing the role of LTI verges as habitat and/or corridor for biodiversity is of importance as verges might contribute to ecological networks. In the last decades, ecological networks of terrestrial and aquatic continuities (blue-green infrastructures) aiming to decrease fragmentation have received much attention from scientists and policymakers [16]. A meta-analysis of corridor effectiveness showed that, overall, corridors increase movements of plants, vertebrates and invertebrates between habitat patches, but that corridor effectiveness varies among taxa [17]. Maintaining such a network of ecological corridors might be beneficial on the long term in the context of climate change by facilitating species dispersal to newly suitable areas [18]. In France, the concept of green and blue infrastructures led to the development of a public policy named "Trame Verte et Bleue" (meaning green and blue ecological network) launched by the French Ministry of Ecology in 2007. Accordingly, French administrative regions have identified ecological networks and they conduct action plans for preserving and restoring these continuities, for biodiversity. At a smaller spatial scale, i.e. townships, it has also to be considered in local urban planning.

\section{Topic identification and stakeholder input}

In France, the LTI network is very dense. For instance, the road network is the longest (over a million kilometres long, $1 / 4$ of the European network) and one of the densest $\left(1.77 \mathrm{~km} / \mathrm{km}^{2}\right)$ of the European Union. As a comparison, Spain, which has an area close to the one of France, has a road density six times lower $\left(0.32 \mathrm{~km} / \mathrm{km}^{2}\right)$. The railway network is also one of the longest in Europe with more than $30,000 \mathrm{~km}$ of railway lines in use. Thus, such a dense LTI network means a considerable inherent surface of verges and LTI managers might substantially contribute to ecological networks. Furthermore, in the literature, 
the potential for LTI verges to be a habitat and/or a corridor for biodiversity seems to exist, but a comprehensive understanding of the conditions where this is true remains, as far as we know, unavailable. Seminal reviews on this question were previously published $[8,14,19]$ but they focused on roads and do not fulfil the standards of a systematic review [20]. This situation motivated several French LTI managing companies and the French Ministry of Ecology to request a systematic review on this issue, at the heart of the green and blue infrastructure public policy. The French LTI managing companies are gathered in an informal group, named "Club des Infrastructures Linéaires \& Biodiversité" (CILB), aiming at acting for biodiversity conservation. The motorway, railway, power line, pipeline and waterway French stakeholder companies who are members of the CILB were specifically interested in evaluating whether their LTI verges could contribute to green and blue infrastructures to improve the management of these verges for that purpose. The systematic review was assumed to be a relevant scientific method to provide a sound answer to this practical questioning from LTI managers. A call for tender for a systematic review was thus launched by the French Ministry of Ecology and the French Agency for Environment and Energy Management (ADEME) through its research incentive program related to transportation ecology, named "Infrastructures de Transport Terrestre, Écosystèmes et Paysage" (ITTECOP), supported by the CILB and the "Fondation pour la Recherche sur la Biodiversité" (FRB), a French foundation supporting research in biodiversity. The French National Museum of National History (MNHN) was then chosen for conducting the project, in collaboration with the French National Research Institute of Science and Technology for Environment and Agriculture (IRSTEA), the University Pierre and Marie Curie (UPMC, Paris 6), the French Centre for Studies and Expertise on Risks, Environment, Mobility, and Urban and Country Planning (Cerema), and the French National Institute for Agricultural Research (INRA). At the beginning of the project, the LTI managers funding the study were met to list the types of verges they own and the management practices they apply on those, to define the components of the review question.

The protocol of the systematic review was published in 2016 [21]. Because the question encompasses all biodiversity, a very large number of articles were collected. The review process was thus split by taxa and a first systematic review focusing on insects was published in 2018 [22]. In particular, this review revealed that insect abundance was generally not statistically different between LTI verges and away from LTIs. Insect abundance was even higher on non-highway road verges than away from roads. Similarly, a recent study showed that road verges and their associated hedges can provide hotspots of resource for pollinators in agricultural landscapes, but their capacity to do so is reduced by heavy traffic (like on highways) [23]. The aim of the present paper was to complement the previous work on insects published by Villemey et al. [22], focusing this time on vertebrates (mammals, birds, amphibians and reptiles). Organisms like fishes that are not living on or using verges but exclusively live in or use the LTI itself were not considered in this review.

\section{Objective of the review}

The primary aim of this review was to assess whether LTI verges can provide habitats for vertebrates (mammals, birds, amphibians and reptiles) and whether they can be used as movement corridors by these taxa. The review exclusively focused on the longitudinal effect of LTI verges on vertebrate (excluding fishes) diversity and movements and did not consider the transversal effects of LTIs such as barrier effects. The review also aims at assessing the effect of management practices (e.g. mowing), as well as of the characteristics of the surrounding landscape, on the potential of LTI verges for vertebrate biodiversity.

\section{Primary question}

The primary question of the review is: can linear transportation infrastructure verges constitute habitats and/or corridors for vertebrates in temperate ecosystems?

\section{Secondary questions}

We split the above primary question of the review into six more specific questions detailed in Table 1 . This subdivision was used during study validity assessment and the synthesis of evidence.

We defined LTI verges as the area up to $30 \mathrm{~m}$ from roadways, waterways, or railways, or the area (whatever the width) below power lines or below/above pipelines. We considered the surrounding landscape to be at, at least, $1 \mathrm{~km}$ around the LTI.

\section{Components of the primary question}

Population: All vertebrate (except fishes) species and communities of the temperate climatic zone.

Exposure: LTI verges (road, railway, power line and pipeline verges and waterway banks). As our systematic review focused on LTIs we only considered verges of navigable waterways (navigable rivers and canals) as relevant exposures. 
Table 1 Details of the six specific questions of the review

\begin{tabular}{ll}
\hline Number & Details \\
\hline Question Q1 & Do LTI verge management practices increase, decrease or have no effect on vertebrate biodiversity in LTI verges? \\
Question Q2 & Is vertebrate biodiversity in LTI verges equal to, higher, or lower than in similar habitats away from LTIs? \\
Question Q3 & Do LTI verge management practices increase, decrease or have no effect on vertebrate movements in LTI verges? \\
Question Q4 & Are vertebrate movements in LTI verges equal to, higher, or lower than their movements in similar habitats away from LTIs? \\
Question Q5 & Is vertebrate biodiversity in LTI verges dependent on the surrounding landscape? \\
Question Q6 & Are vertebrate movements in LTI verges dependent on the surrounding landscape?
\end{tabular}

Intervention: Management practices (e.g. mowing) or human-induced disturbances (e.g. waterway channelization) on LTI verges.

Comparator: Both temporal and spatial comparators, including but not restricted to temporal comparators (ecosystem present before versus after infrastructure construction (LTI verge creation), LTI verge before versus after management intervention) or spatial comparators (LTI verges versus nearby similar habitats away from LTIs, LTI verges managed with one practice versus unmanaged LTI verges or LTI verges managed with a different practice).

Outcomes: All outcomes relating to species presence or species movements, including but not restricted to species richness, abundance, community composition and species dispersal movements.

Context: Because the funders requested an evidence synthesis applicable to western Europe, we restricted our synthesis to temperate zones.

\section{Methods}

The methods are described in detail in an a priori systematic review protocol [21]. We summarize it here and present the small deviations from this protocol that we made when conducting the review. The methods follow the Collaboration for Environmental Evidence (CEE) Guidelines and Standards for Evidence Synthesis in Environmental Management [24] unless noted otherwise, and this paper conforms to ROSES reporting standards [25] (see Additional file 1).

\section{Search for articles Search strings}

The review team identified English search terms to be combined in search strings. For all keywords listed wildcards may be used to allow the use of derivations of the word's root and to account for the possibility of finding a word in various spellings (English from Great Britain or from the United States) and with various endings (singular or plural).

We tested a first search string combining some of the search terms with Boolean operators of Web Of Science
Core Collection (with search on "Topic"). To assess the comprehensiveness of the search string, we compared the search hits to the articles of the test list indexed in the database (see Additional file 2 for the list of articles of the test list and how it was constituted). Then, we modified the search string by removing some of the search terms and including new ones, to increase the number of articles of the test list retrieved [21]. At last, the search string that produced the highest efficiency (i.e. total number of search hits as low as possible with the highest number of articles from the test list retrieved) was a set of four subsearch strings displayed in Table 2.

\section{Publication databases}

We first listed the databases to which the members of our review team had access. The database selection was then based on three criteria [21]:

- Topic: the database(s) had to cover ecology;

- Accessibility/reproducibility/sustainability: the database(s) had to be accessible by the whole review team, and by researchers all over the world (as a guarantee of reproducibility and further reviewing);

- Comprehensiveness: number of articles indexed in the database(s) among the articles of the test list (Additional file 2).

These criteria led us to select two databases: Web Of Science Core Collection (with subscriptions: Science Citation Index Expanded 1956-present, Social Sciences Citation Index 1975-present, Arts and Humanities Citation Index 1975-present, Conference Proceedings Citation Index-Science 1990-present, Conference Proceedings Citation Index-Social Science \& Humanities 1990-present, Book Citation Index-Science 2005-present, Book Citation Index-Social Sciences and Humanities 2005-present, Emerging Sources Citation Index 2015-present, Current Chemical Reactions 1985-present, and Index Chemicus 1993-present; 84 articles indexed out of the 102 articles of the test list) and Zoological Records (subscribed timespan 1864-present, 51 articles 
Table 2 Sub-search strings selected and used in Web Of Science Core Collection and Zoological Records publications databases

\begin{tabular}{|c|c|c|}
\hline LTI & Strategy & Search string \\
\hline \multirow[t]{2}{*}{$\begin{array}{l}\text { Roads, railways, } \\
\text { pipelines and } \\
\text { powerlines }\end{array}$} & 1 & $\begin{array}{l}\text { LTIs: ("transport* infrastructure*" OR road* OR highway\$ OR motorway\$ OR freeway\$ OR rai** OR pipeline\$ OR pow- } \\
\text { erline\$ OR "power line" OR "power lines" OR "transmission line*" OR "electric line" OR "electric* lines" OR "electric* } \\
\text { pylon*") } \\
\text { AND } \\
\text { Verges/outcomes: (corridor\$ OR dispersal\$ OR habitat\$ OR refuge\$ OR "right* of way*" OR verge\$ OR abundance OR } \\
\text { richness OR composition\$ OR *diversity OR communit*) }\end{array}$ \\
\hline & 2 & $\begin{array}{l}\text { LTIs: (road* OR highway* OR motorway* OR rail* OR"transmission line* corridor*" OR powerline* OR pipeline* OR } \\
\text { "electric* pylon*") } \\
\text { AND } \\
\text { Verges: (corridor* OR habitat* OR verge* OR right\$-of-way* OR proximity OR contiguous OR line\$) } \\
\text { AND } \\
\text { Outcomes: (dispers* OR population* OR communit* OR abundan* OR distribution\$ OR "species composition*" OR } \\
\text { attendance) }\end{array}$ \\
\hline \multirow[t]{2}{*}{ Waterways } & 1 & $\begin{array}{l}\text { LTIs/verges: (riparian OR riverside\$ OR riverbank\$ OR “river* *bank*" OR ((waterway\$ OR canal\$ OR channel\$) } \\
\text { AND *bank*)) } \\
\text { AND } \\
\text { Outcomes: (corridor\$ OR dispersal\$ OR habitat\$ OR refuge\$ OR abundance OR richness OR *diversity OR composi- } \\
\text { tion\$ OR communit*) }\end{array}$ \\
\hline & 2 & $\begin{array}{l}\text { LTIs: (river* OR channel\$ OR stream\$) } \\
\text { AND } \\
\text { Verges: (riparian\$ OR *bank* OR proximity OR bridge\$) } \\
\text { AND } \\
\text { Outcomes: (dispers* OR communit* OR richness OR diversity OR drowning OR roosting OR “alien plant*") }\end{array}$ \\
\hline
\end{tabular}

The asterisk (*) represents any group of characters, including no character. The dollar sign (\$) represents zero or one character. The quotation marks ("'") ) allow to look for an exact phrase. Strategies 1 and 2 are explained in [21]

out of the 102 articles). Searches on these two databases were made on "Topic".

\section{Search engines}

We performed additional searches using three search engines:

- Google Scholar (https://scholar.google.fr/);

- BASE (Bielefeld Academic Search Engine, https:// www.base-search.net/);

- CORE (https://core.ac.uk/).

Because these search engines could only handle a limited number of search terms and did not allow the use of all wildcards, the search strings used for publication databases were simplified. We thus developed a search string for each of the five LTIs (Additional file 3). In Google Scholar, results were sorted by relevance, with the boxes "include patents" and "include citations" unchecked. In BASE, results were sorted by relevance, with the box "boost open access documents" unchecked and the box "Verbatim search" checked. For each of the five search strings, we retrieved the first 20 hits.

\section{Specialist websites}

We searched for links or references to relevant articles and data on 11 specialist websites including a journal special issue on transportation ecology (Additional file 4).

\section{Supplementary searches}

To retrieve grey literature, we contacted by email national and international experts of transportation ecology, through the Ecodiff (now SFEcodiff), Transenviro, Wftlistserv and IENE mailing lists and by posting a call on social media (https://fr.linkedin.com/). SFEcodiff is a French mailing list about ecology and evolution which counted around 7000 subscribers (https://www.sfeco logie.org/sfecodiff/), and Transenviro, Wftlistserv and IENE mailing lists are international mailing lists about transportation ecology. Together, the Transenviro and Wftlistserv mailing lists (http://www.itre.ncsu.edu/CTE/ Lists/index.asp) gathered about 600 contacts and the IENE mailing list (http://www.iene.info/) counted around 300 contacts. All these mailing lists were accessed on 22 September 2015. Eventually, we contacted nearly two thousand people $(\mathrm{N}=1902)$ by individual email. Organizations funding the systematic review also provided us with their unpublished reports. 


\section{Dates of literature searches}

Literature searches were performed in two stages. First, we performed searches in Web Of Science Core Collection publication database, in Zoological Records publication database, and in Google Scholar search engine on April 27th 2015, February 1st 2016, and March 4th to 9th 2016, respectively. The call for grey literature was performed on April 21st 2015. All articles published in 2016 were not considered during these first searches. Second, searches were updated for articles published from 2016 onward, on June 15th 2018 for Web Of Science Core Collection and Zoological Records publication databases, and on November 6th 2018 for Google Scholar (Additional file 4). The call for grey literature was updated for articles published from 2016 onward through searches on specialist websites that were conducted from November 26th 2018 to December 4th 2018, and searches on BASE and CORE search engines that were performed on November 7th and 8th 2018, respectively.

\section{Article screening and study eligibility criteria Screening process}

The articles collected from online publication databases were screened by several members of the review team for eligibility (according to the criteria described in the next section) through three successive stages: first on titles (performed by AJ, EG, ELM, MV, RS, SV and VR), second on abstracts (performed by AC, AJ, EG, ELM, MV, RS, SV, VR and YB) and third on full-texts. The screening is conservative at each stage, i.e. in cases of doubt, articles proceed to the next stage for further assessment. The agreement between screeners was assessed before beginning the screening on titles and abstracts by computing a Randolph's Kappa coefficient [21] on a number of references randomly sampled among the sets of articles about waterways and other LTIs (200/62,940 titles and 20/23,588 abstracts), and screened by each of the reviewers independently of each other. We considered 200 and 20 randomly sampled references to be sufficient to assess the agreement between screeners during title and abstract screening, respectively. It is a relatively small proportion of the total number of references to be screened, but these numbers were based on our experience with the first systematic review published on insects [22], and the review team was nearly identical for this review on vertebrates. A minimal coefficient of 0.6 was considered an acceptable level of agreement between reviewers. All disagreements were discussed by reviewers, so that differences in screeners' understanding of eligibility criteria could be resolved. When the coefficient was lower than 0.6 the operation was repeated until reaching a coefficient larger than 0.6.
Screening on full-texts was performed by AC, AJ, AV, DYO, EG, ELM, MV, RS, SV, VR and YB. Although this is a good practice to assess the agreement between screeners at full-text screening we were unable to do this due to logistical constraints and time limitation. We however performed a retrospective check of a random sample (10\%) of all the articles rejected. Because one screener (DYO) screened a large proportion of full-texts (41\%), cross checking was performed as follows: one screener (RS) checked $10 \%$ of the articles rejected by DYO, and DYO checked $10 \%$ of the articles rejected by all other screeners. Decisions appeared consistent for $91 \%$ of the articles. For articles with inconsistent decisions, they were finally excluded after discussion.

The articles collected from search engines, call for literature, and searches on specialist websites were screened on titles and then directly on full-texts.

During the screening process reviewers did not screen articles they themselves had authored.

\section{Eligibility criteria}

At each stage of screening, article eligibility was based on a list of selection criteria. At the stage of title screening, these criteria mainly encompassed both the subject (ecology and related disciplines) and the population and exposure/intervention of the article (Table 3). The same criteria were applied at the stage of abstract screening, to which we added criteria regarding the exposure/intervention, the comparator, the outcomes or the study type (Table 4). Articles without abstract were discarded due to their high number and time constraints, although we acknowledge that CEE guidelines recommend that their eligibility should be assessed at full-text screening. Finally, the same criteria as for the abstract stage were used for the stage of full-text screening, to which we added new inclusion criteria regarding the language, the climate, the type of publication or the specific questions covered (Table 5). We considered that a study was not relevant to the purpose of the review, and thus discarded it, if the comparator was inappropriate [comparison between different seasons, comparator difficult to interpret for the purposes of this review, high contrast of habitat with the comparator (e.g. herbaceous vegetation compared to forest), etc.], if the sampling was not strictly done on verges [we defined LTI verges as the area up to $30 \mathrm{~m}$ from roadways, waterways, or railways, or the area (whatever the width) below power lines or below/above pipelines], or if-for questions Q5 and Q6-the landscape scale was below $1 \mathrm{~km}$. As our review focused on transportation infrastructures, we also made sure at full-text screening stage that only paved roads and navigable rivers and canals were included. This information is unfortunately rarely provided for waterways, so we included all articles with Strahler [26] stream 
Table 3 List of eligibility criteria at the stage of title screening

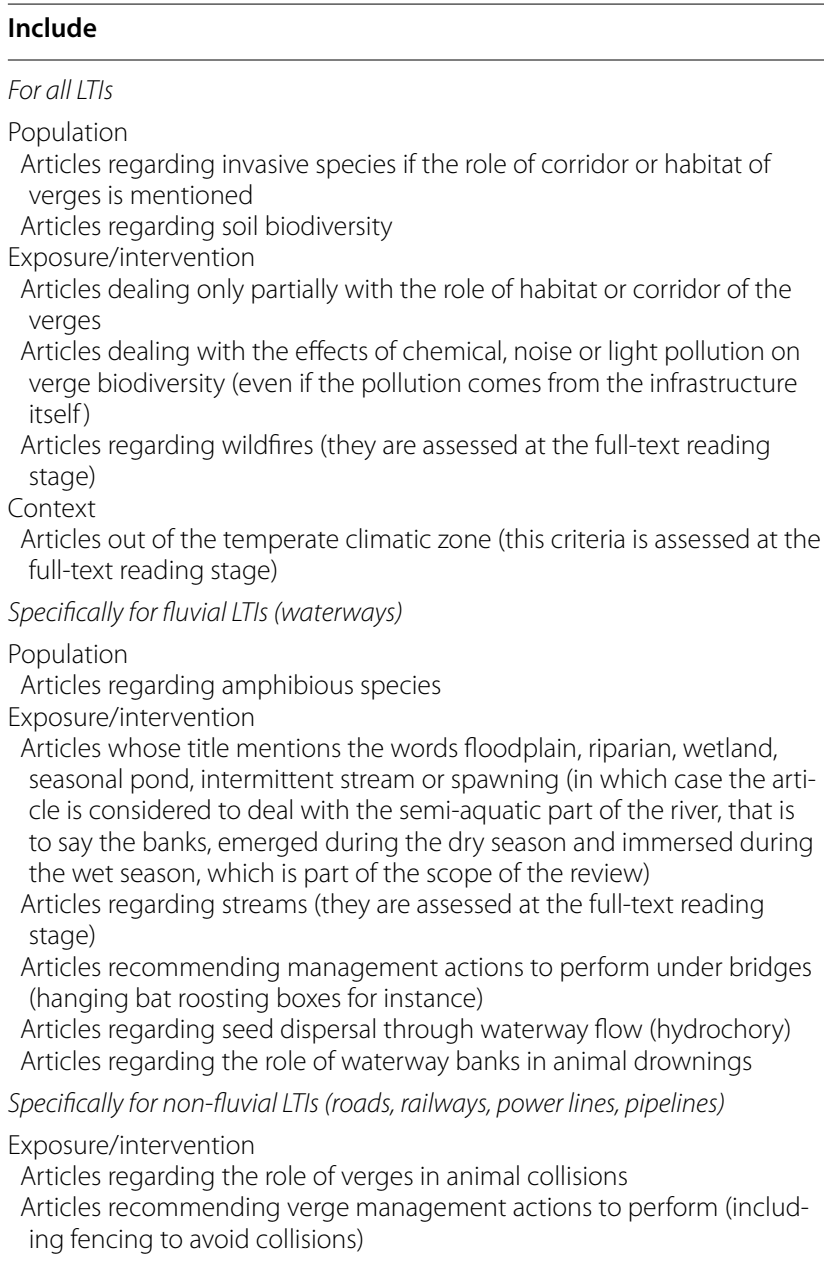

\section{Exclude}

Population

Studies regarding paleontology, phylogenetics, phylogeography and taxonomy (including studies describing newly discovered species) Genetic studies without any relation to a natural habitat (in particular biodiversity meta-genomics studies)

Pedological studies without any relation to biodiversity

Exposure/intervention

Studies regarding overpasses/underpasses or fragmentation due to LTIS considered transversally, without considering the roles of habitat and corridor of verges

Outcome

Studies regarding green infrastructures in general without considering the specific case of LTIs

Studies regarding toxicology or chemical, noise or light pollution without any relation to biodiversity

\section{Population}

Articles regarding exclusively aquatic species, except if the title mentions the words floodplain, riparian, wetland, seasonal pond, intermittent stream or spawning (in which case the article is considered to deal with the lateral part of the river, that is to say the banks, sometimes immersed other times emerged, which is part of the scope of the review) Exposure/intervention

Articles regarding lakes and islands or sand banks in the middle of rivers Articles regarding river debris (organic matter, tree trunks, underwater leaves decomposition, except if the article deals with the submerged part of the bank, etc.)

Articles regarding drownings without any relation to the role of habitat of the banks

\section{Exposure/intervention}

Articles regarding animal collisions without any relation with the role of habitat or corridor of the verges

\section{Table 4 List of inclusion criteria at the stage of abstract screening}

\begin{tabular}{ll}
\hline Type of criteria & Description \\
\hline $\begin{array}{l}\text { Relevant population(s) } \\
\text { Types of exposure/intervention }\end{array}$ & $\begin{array}{c}\text { All vertebrate biodiversity (at the species, community and ecosystem level), including exotic invasive species } \\
\text { verge management (mowing, pesticide spreading, pruning, planting, fence laying, beehive setting up, bird nesting } \\
\text { box and bat roosting box hanging, reptile artificial refuge setting up, etc.) or to a LTI verge disturbance (chemical, air, } \\
\text { noise and light pollution and wildfires) }\end{array}$ \\
$\begin{array}{l}\text { Unexposed/intervention-free control site or before-exposure/before-intervention control site } \\
\text { Types of outcome }\end{array}$ & $\begin{array}{c}\text { All outcomes relating to corridor and habitat assessment or effects of verge management, such as dispersal (including } \\
\text { species invasions, hydrochory and seed dispersal by vehicles), species richness, Shannon index, Simpson index, beta } \\
\text { diversity, community composition and abundance of different taxonomic or functional groups of organisms } \\
\text { All type of studies should be included apart from modelling (theoretical) articles, articles making recommendations } \\
\text { without making experimentation and articles making experimentations in laboratory conditions }\end{array}$ \\
\hline
\end{tabular}

order above three, canals and rivers, and we excluded all articles with stream order equal or below three and articles with no information on stream order. To identify whether study area was in the temperate climate we used the Köppen-Geiger Climate Classification [Cfa, Cfb, Cfc, Csa, Csb, Csc, see http://people.eng.unimelb.edu. $\mathrm{au} / \mathrm{mpeel} /$ koppen.html (accessed 17 November 2015) for the GoogleEarth layers of the Köppen-Geiger Climate 
Table 5 List of inclusion criteria at the stage of full-text screening

\begin{tabular}{ll}
\hline Type of criteria & Description \\
\hline Language & Full text written in English or French \\
Climate & Articles with study zone(s) of the temperate climate \\
Type of publication & Articles different from editorial material, meeting abstracts, news items and review \\
Comparator & Articles with control/compared site (not observational studies) \\
Road type & Articles with paved road (not unpaved road, path, gravel road, forest road) \\
Waterway type & Articles with stream order above three, canals or rivers \\
Specific questions & Articles that give relevant results to answer one or more of the six specific synthe- \\
& sis questions detailed in Table 1
\end{tabular}

Classification]. When a study area overlapped temperate and non-temperate climate with no possibility to extract the data regarding only the temperate climate the study was discarded. Similarly, studies were excluded if the results included biological groups and/or exposures that were not under the scope of the review, with no possibility to extract results scoping the review (e.g. results combining vertebrates and non-vertebrate species, results combining paths and paved roads). We also checked for data redundancy (data already published in another article included in the review) and added this factor as an exclusion cause.

Articles about non-vertebrate species were set aside during the three stages of title, abstract and full-text screenings.

\section{Study validity assessment}

We conducted a critical appraisal of the studies and assigned them a low, medium or high risk of bias. To define the criteria of this appraisal, eight external experts in landscape connectivity and transportation ecology were gathered and consulted during a 1-day workshop with seven scientists of our review team [21]. During the workshop, we discussed about the gold standard protocol of an ideal study answering our primary question with unlimited resources (unlimited money, time, workforce, etc.). We considered that a study was unreliable because of a high risk of bias, and therefore excluded it from the review, if there was/were:

- A total absence of replications;

- An inadequate methodology (for example for question Q4 on the role of corridor of verges, a statistical analysis of movement data that did not allow to distinguish LTI verges from other habitats);

- A method description strongly insufficient (i.e. when it was not possible to know where the sampling was done: within or outside LTI verges);

- Major confounding factors (e.g. strong difference in sampling effort between treatment and control).
We considered that a study had a medium risk of bias if it had the following characteristics:

- Absence of transparent and systematic procedure for the selection of sample plot location (i.e. randomization, fixed distances, grids);

- Control-Intervention and Before-After-Intervention study designs (as opposed to Before-AfterControl-Intervention study designs) for the specific questions involving verge management (questions Q1 and Q3);

- Absence of true spatial replication of the study (for example study with repetition of measures on a unique site);

- Attrition bias (difference in the loss of samples between control and treatment);

- Method description slightly insufficient (some minor details were missing but did not challenge our understanding of the methods).

Finally, we considered that a study that did not have a high or medium risk of bias had a low risk of bias. Studies with a high risk of bias were discarded from synthesis. In the narrative synthesis, the results of studies with a low risk of bias were first synthesized and then the consistency of the results of studies with a medium risk of bias was assessed. In the meta-analyses, the influence of the level of bias (low or medium) on effect sizes was furthermore tested.

For articles dealing with more than one specific question (Table 1), we performed critical appraisal for each question separately, that we considered being different studies. The critical appraisal was performed as follows: first, each study was critically appraised by one reviewer (DYO, MH or VA). Then, a second reviewer (DYO or $\mathrm{MH}$ ) critically appraised again the uncertain cases. We compared conclusions of the two reviewers, and when they differed, they discussed disagreements until reaching a consensus and asked for a third reviewer if necessary. All reviewers never had to critically appraise articles 
they authored by themselves. Although it is a CEE standard that at least two people independently critically appraise each study, it was not possible in this study due to the high number of articles and time constraints.

\section{Data coding and extraction strategy Extraction of meta-data}

We used the coding tool displayed in Table 6 to produce an easily searchable database of the studies included after critical appraisal (i.e. with low and medium risk of bias). If an article dealt with more than one of our specific questions, we coded each question in a different row.

\section{Extraction of data for narrative syntheses}

For all specific questions, we first extracted into tables the statistically tested results of all studies with low and medium bias. For each species or group of species we extracted the effects of exposure/intervention and categorized them as positive, negative or neutral. Neutral effects referred to comparison between control and treatment that were statistically not significant (i.e. no statistically significant difference between the two, $\alpha=0.05$ ). Where necessary, we assessed whether the differences were statistically significant using the confidence intervals reported by the authors. Data extraction was performed by one reviewer (DYO) and a second reviewer
(AC) checked all the studies related to the specific questions Q1, Q4, and Q5.

\section{Extraction of data for meta-analyses}

For each primary study, and for both LTI verges and control sites away from LTIs, sample sizes, outcome means, and measures of variation (standard deviation, standard error, or confidence interval) were extracted from tables, text, published raw data (e.g. in appendices), and graphics using WebPlotDigitizer version 4.2 (https://autom eris.io/WebPlotDigitizer, accessed 17 June 2019). When outcome means or measures of variation could not be directly extracted from the published data, the sample size and any other measure that enable further imputation according to Lajeunesse [27] (e.g. upper- and lower inter-quartile ranges, statistical tests parameters) were extracted. In case of uncertainty of the measure of variation reported (i.e. when it was impossible to know whether it was the standard deviation or the standard error that was reported), authors were directly contacted to recover missing information. When outcome means and measures of variation were available on a log-scale basis, they were back-transformed according to Higgins et al. [28]. Abundance for either species groups or individual species were extracted. If a study reported the abundances for both a group and some particular individual species from the group we only used the former.

\section{Table 6 Coding tool for the database of included studies}

\begin{tabular}{|c|c|}
\hline Coding variable & Details/Examples \\
\hline ID & Unique identifier of the publication \\
\hline Source & Source of the publication (e.g. WOS, ZR, grey literature) \\
\hline Reference & Authors, year, title, publisher \\
\hline \multicolumn{2}{|l|}{$\mathrm{DOI}$} \\
\hline Publication type & Book chapter, journal article, thesis, report, etc. \\
\hline Article language & English/French \\
\hline Specific question & Question Q1, Q2, Q3, Q4, Q5, or Q6 \\
\hline Study design & Spatial/temporal/spatial and temporal comparisons \\
\hline Risk of bias & Low/medium \\
\hline \multicolumn{2}{|l|}{ Study country } \\
\hline \multicolumn{2}{|l|}{ Study region(s) } \\
\hline \multicolumn{2}{|l|}{ GPS coordinates } \\
\hline Biological group(s) & Amphibians/birds/reptiles/small mammals/bats/other mammals \\
\hline $\mathrm{LTI}$ & Roads/railways/powerlines/pipelines/waterways \\
\hline LTI verge & Description of verge and its habitat (grassland, shrubland, hedge, forest, etc.) \\
\hline Comparison & $\begin{array}{l}\text { Questions Q2 and Q4: type of habitat of the control site } \\
\text { Question Q1 and Q3: management practices (mowing, pesticide spreading, } \\
\text { pruning, planting, fence laying, etc.) } \\
\text { Questions Q5 and Q6: landscape metric(s) and spatial scale(s) }\end{array}$ \\
\hline Outcomes & Abundance, species richness, Shannon index, etc. \\
\hline \multicolumn{2}{|l|}{ Included in narrative synthesis } \\
\hline Included in meta-analyses & \\
\hline
\end{tabular}


Ten studies measured the biodiversity of vertebrates at various distances from LTI verges [29-38]. In these cases, we used values of the furthest distance as controls. Finally, if a study reported several sites that could serve as a control, the site with habitat most similar to LTI verges was chosen as control. Data extraction was performed by one reviewer (DYO) and a sample of data (60\%) was retrospectively cross checked by another reviewer (YR).

\section{Potential effect modifiers/reasons for heterogeneity}

We recorded the following potential effect modifiers as stated in the protocol of the present review [21]:

- Geographic location;

- Biological group of vertebrates studied;

- Site characteristics: type of LTI, type of habitat of the verge and type of habitat of the compared site;

- Verge management practices (mowing, grazing, vegetation burning, pesticide use, etc.);

- Comparator type (spatial/temporal, etc.);

- Selection of sampling location (randomization, fixed distances or grids versus directed sampling).

Although identified as a potential reason for heterogeneity in the protocol of the present review [21], we eventually considered the absence of replicates as an important source of bias. Accordingly, those articles without replicates were discarded during critical appraisal.

\section{Data synthesis and presentation}

\section{Descriptive statistics and narrative synthesis}

The meta-data extracted from each study were used to produce descriptive statistics of the evidence. Then, for
To be included in the meta-analyses, studies had to report mean, sample size and some measure of variation for vertebrate abundance or species richness, for both LTI verges and another habitat away from the LTI that served as a control (in addition to the inclusion criteria used for the whole review). When we could not get some measure of variation from primary studies they were estimated via data imputation using the available means and standard deviations of all the studies with complete information [27], and when we could not get means we directly estimated effect sizes (Hedges' $d$ standardized mean difference, see next section) from statistical tests parameters (e.g. from t-value of t-test) [27].

In the meta-analyses, we used as response variables abundance and its proxies (density, number of captures, of passes, of contacts, of calls, of litters, of nests, of breeding pairs, of pellets, or of territories), and species richness.

\section{Meta-analyses}

To assess the response of vertebrates to LTI (specific question Q2) we used the Hedges' $d$ standardized mean difference [39] as a measure of the effect size for both abundance and species richness:

$$
d_{i}=\frac{\bar{X}_{i, \text { treatment }}-\bar{X}_{i, \text { control }}}{S_{\text {pooled }_{i}}} * J_{i}
$$

where $\bar{X}_{i, \text { treatment }}$ and $\bar{X}_{i, \text { control }}$ are the means for study $i$ of treatment sites on LTI verges and control sites away from LTI, respectively. Thus, the effect size $d_{i}$ is positive if the abundance or species richness is higher in LTI verges than in sites away from LTI.

$S_{\text {pooled }_{i}}$ is the pooled standard deviation of the two groups:

$$
S_{\text {pooled }_{i}}=\sqrt{\frac{\left(n_{i, \text { treatment }}-1\right) * S D_{i, \text { treatment }}^{2}+\left(n_{i, \text { control }}-1\right) * S D_{i, \text { control }}^{2}}{n_{i, \text { treatment }}+n_{i, \text { control }}-2}}
$$

each specific question, we produced a narrative table that summarized the key results of relevant studies and we wrote a narrative synthesis. Whenever possible, we organized the findings from included studies by grouping them into categories based on risk of bias, biological group, type of LTI, and/or type of management intervention.

\section{Eligibility for meta-analysis}

Meta-analyses were only possible for the specific question Q2 (role of habitat of LTI verges) because only this question gathered enough homogeneous studies in terms of comparator and outcome with the required statistics. where $S D_{i}$ is the standard deviation and $n_{i, \text { treatment }}$ and $n_{i, \text { control }}$ are the sample sizes of treatment and control groups.

$J_{i}$ is a correction for small sample size:

$$
J_{i}=1-\frac{3}{4 *\left(n_{i, \text { treatment }}+n_{i, \text { control }}-2\right)-1} .
$$

To calculate the variance for Hedges' $d_{i}$ we did not use the standard approach with Hedges' estimator [40] because Hamman et al. [41] demonstrated that it is biased under conditions common in ecological metaanalyses. Instead we used the alternative estimator proposed by Hedges [42]: 


$$
\operatorname{var}_{i}=\left(1-\frac{3}{4 *\left(n_{i, \text { treatment }}+n_{i, \text { control }}-2\right)-1}\right)^{2} *\left(\frac{n_{i, \text { treatment }}+n_{i, \text { control }}-2}{\frac{n_{i, \text { treatment }} * n_{i, \text { control }}}{n_{i, \text { treatment }}+n_{i, \text { control }}}\left(n_{i, \text { treatment }}+n_{i, \text { control }}-4\right)}\right)
$$

We used linear mixed-models with the restricted maximum-likelihood (REML) estimator to estimate the grand mean effect size and test the effect of moderators. Because one study could gather several cases, we nested the cases within the studies as random effects for each analysis. A case referred to an individual effect size extracted from a study (e.g. abundance of a particular species or group of species); a given study possibly gathering multiple cases (e.g. abundance of several species or group of species).

In the models each effect size was weighted based on the precision of its estimate, with more precise estimates receiving greater weights. Weights $w_{i}$ were computed as $w_{i}=1 /\left(\operatorname{var}_{i}+\tau^{2}\right)$ with $\tau^{2}$ the among-study variance estimated during the meta-analysis.

For each response variable (i.e. abundance and species richness), we first computed the grand mean effect size combining all studies (mixed model without moderators with cases nested within studies as random effects). We analysed the datasets for publication bias (i.e. when the published literature reports results that systematically differ from those of all studies conducted) using funnel plots, Pearson's correlation coefficient between effect size and publication year, cumulative meta-analysis by publication year, and Rosenberg's fail-safe number [43]. Publication bias is manifested by an asymmetry in funnel plot, but other causes than publication bias can lead to funnel plot asymmetry, such as heterogeneity in effect sizes. To take this into account, we used the residuals of the model testing the effect of the type of LTI on vertebrate response to graphically examine and test for funnel plot asymmetry [44] using Egger's regression test [45]. At this stage we also tested for the effect of the risk of bias of the studies (low or medium). Because the risk of bias was correlated with the type of LTI (e.g. all studies on powerlines had a low risk of bias whereas all studies on railways had a medium risk of bias) and thus the effect of risk of bias may be confounded with the effect of type of LTI, we tested the effect of the risk of bias on the residuals of the model testing the effect of the type of LTI on vertebrate response.

We investigated in meta-analyses the influence of LTI type and biological group (moderators) on vertebrate response to LTI. We evaluated vertebrate response to six LTI types: highways, non-highway roads, waterways, pipelines, powerlines, railways. We discriminated between highways from other roads because road width, verge width and disturbances (traffic, noise, light, pollution) can vary considerably between the two types. To categorize highways, we used the description given by the authors of the publication and considered "highway", "motorway", "freeway", and "6-lane roads" as highways. We evaluated the heterogeneity in vertebrate response to LTIs by assessing response variation with biological group. We defined six biological groups: amphibians, reptiles, birds, small mammals (less than $1 \mathrm{~kg}$ rodents, shrews), bats, and other mammals.

For testing the effects of moderators, we avoided problems associated with confounding factors by constructing independent subsets of data. We also restricted our analysis to variable levels that were represented in at least two studies. We also determined the influence of individual cases on the results by computing Cook's distance, and we removed from analysis the cases with a distance greater than 0.5 . As a result of these restrictions, we were able to analyse the effect of biological group on vertebrate abundance for each LTI separately (i.e. one analysis per LTI); and conversely, the effect of LTI on vertebrate abundance for each biological group, which was the other possible option to split the data. We checked the consistency of the results of these two options. Regarding species richness, we were able to analyse bird species richness for three LTIs.

In all analyses total heterogeneity $Q_{T}$ was partitioned into heterogeneity explained by the model $\left(Q_{M}\right)$ and heterogeneity not explained by the model $\left(Q_{E}\right)$ with $Q_{T}=Q_{M}+Q_{E}$. The statistical significance of $Q_{M}$ and $Q_{E}$ was tested against a $\chi^{2}$ distribution.

All analyses were conducted in $\mathrm{R}$ 3.5.3 [46] using the metafor package [47] and the rma.mv() function.

\section{Deviations from the protocol}

Primary question:

- The question in the protocol originally encompasses all biodiversity. Because a very large number of articles were collected, the review process was split by taxa and the primary question in this review focuses on vertebrates.

Search for articles:

- Searches on search engines were performed using search terms in English only, and not in both English and French. 
- Searches on Google (https://www.google.fr) were not conducted.

- Searches on specialist websites were not performed at the beginning of the review (searches on Google Scholar and the call for literature were considered sufficient). However, the update for grey literature was conducted through searches on specialist websites, as well as through searches on Google Scholar, BASE (https://www.base-search.net/) and CORE (https://core.ac.uk/), and the call for literature was not carried out once more.

\section{Screening:}

- The consistency of reviewers' decision during fulltexts' screening was not tested, due to logistical and time constraints.

- Although it was not clearly stated in the protocol, it is a CEE standard that articles without abstracts are assessed at full-text. However, in this review, articles without abstracts were directly excluded due to their high number and time constraints.

Data extraction:

- For readability, the coding variables "Publication year", "Authors", "Title", and "Publisher" were aggregated into a single column "Reference". For better referencing of the studies, the variable "DOI" was extracted. The unique identifier of the publication, the source of the publication, and the specific question (Q1-Q6) addressed by the study were also indicated in the final table of included studies, as well as whether the study is included in the narrative synthesis and in the meta-analyses.

The implications of these deviations are considered in the review limitations section.

\section{Results}

Literature search, retrieval, screening and appraisal Literature searches and screening

Searches returned 61,412 from Web Of Science Core Collection, and 21,160 from Zoological Records publication databases. We found 83 of the 84 articles of our test list indexed in Web Of Science core collection, and 49 of the 51 articles of our test list indexed in Zoological Records (Additional file 2). Combining the two databases, only one (out of 102) article of our test list was indexed in the databases but not found by our searches. The other searches gave 200 records from Google Scholar, 100 from BASE, 100 from CORE, 98 from specialist websites, and
495 from the 52 persons who answered the call for grey literature.

Eventually, all the searches gave a total of 83,565 individual records which resulted in 62,940 articles after duplicate removal (Fig. 1). Of them, 23,588 remained after title screening, and 3191 after abstract screening. We could not retrieve 209 full-texts, leaving 2982 full-text to screen. At full-text screening the most common reasons for exclusions were: non-temperate climate (46\%), inappropriate comparator (12\%), no intervention/ exposure (11\%), criteria specific to waterways (9\%), and full-text language other than English or French (7\%). After full-text screening 204 articles remained (Fig. 1). The articles rejected at full-text screening as well as those for which we did not find full text are listed in Additional file 5 with reason for exclusion.

\section{Study validity assessment}

Because an article can answer to more than one of our specific questions, the 204 articles included in the review were split into 221 different studies that underwent critical appraisal. At this stage, 93 studies were excluded from further synthesis (Additional file 6, list of the studies included in the review with study validity assessment) because they showed a high risk of bias (but one study was excluded because data was redundant with another study). The reasons for a high risk of bias were the presence of a major confounding factor (53 studies), a strongly insufficient description of the method (30 studies), and an absence of replication (9 studies, Fig. 1). Finally, a total of 128 studies corresponding to 119 articles were retained after critical appraisal (Additional file 7, list of the studies included in the review with coded metadata). To be further included in the narrative syntheses studies must provide statistical results (91 studies, Fig. 1). To be further included in the meta-analyses, studies must provide appropriate data (35 studies, 29 of them were already included in the narrative syntheses). This leaves 31 studies that did not provide statistical results nor appropriate data for meta-analyses. These studies, although included in the review, were therefore neither included in the narrative syntheses nor in the meta-analyses (Additional file 7, Fig. 1).

\section{Characteristics of the studies}

Of the 128 included studies with low or medium risk of bias, only $23 \%$ (30 studies) had a low risk of bias (Fig. 2). Most of the 128 studies dealt with the role of habitat of verges (question Q2) and compared vertebrate biodiversity in verges to that of other similar habitats (82 studies). Twenty-one studies dealt with the effect of verge management on vertebrate biodiversity (question Q1) and 16 
ROSES Flow Diagram for Systematic Reviews. Version 1.0

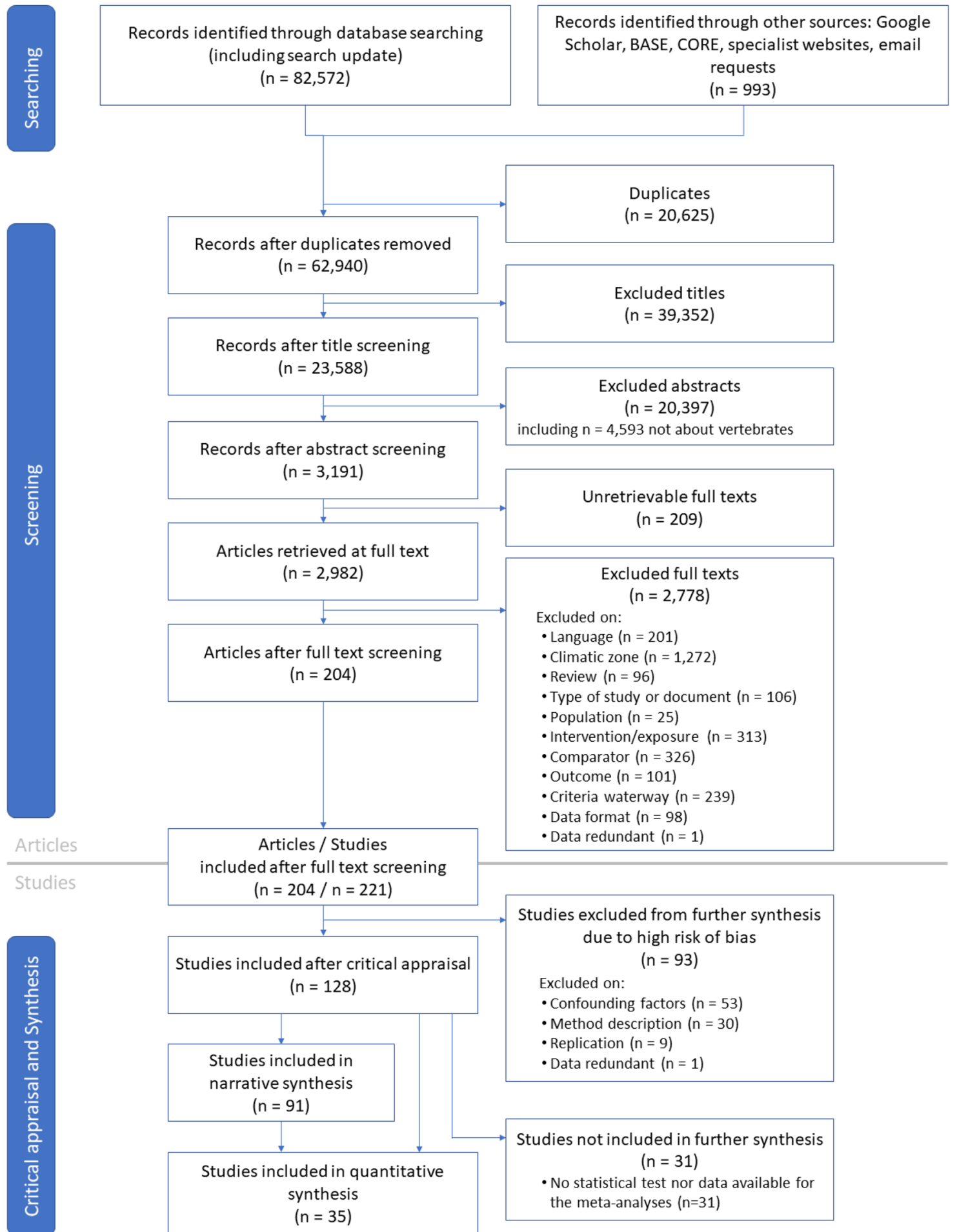

Fig. 1 ROSES flow diagram [25] reporting the screening process of the articles and studies of the review 


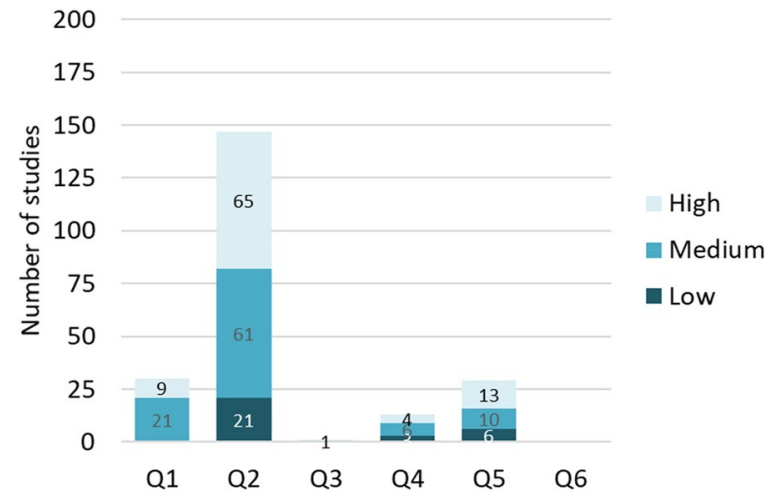

Fig. 2 Number of studies with low, medium and high risk of bias for each specific question. For details on the specific questions see Table 1. No study dealing with the specific question Q6 was found, and only one study with a high risk of bias was found for question Q3

with the influence of the surrounding landscape on the biodiversity hosted by verges (question Q5). We found nine studies that dealt with the role of corridor of verges (question Q4) and compared vertebrate movements in verges to those in another linear habitat away from LTIs. We did not find any studies with low or medium risk of bias dealing with the influence of management or surrounding landscape on the role of corridor of verges (questions Q3 and Q6, respectively).

\section{Publication year}

The 119 articles corresponding to the 128 studies with low or medium risk of bias were published from 1972 to 2018 , with an exponential increase in the number of articles with time (Fig. 3a). The increased number of articles over time is not explained by an increase in the quality of studies over time, as the pattern was similar for the number of articles after and before critical appraisal (Fig. 3b).

\section{Source}

The 119 articles corresponding to the 128 studies with low or medium risk of bias were mainly retrieved from Web Of Science Core Collection (93 articles) and Zoological Records (21) publication databases. The literature sources brought relatively few articles: two articles were sent to us following the call for grey literature, one came from Google Scholar, one was found on a specialist website (journal special issue on transportation ecology), and one was found through a received announcement of defence of thesis.

\section{Language and type of article}

The articles corresponding to the 128 studies with low or medium risk of bias were mainly written in English
(115 out of 119), the others being written in French (articles written in other languages were excluded at fulltext screening). The articles were mainly journal articles (113), but the review also included three book chapters, two Ph.D. thesis chapters, and one technical report.

\section{Study location}

At country level, most of the 128 studies were conducted in the United States of America (22\%, Fig. 4), then in United Kingdom (16\%), Australia (13\%), France (13\%) and Spain (8\%).

\section{Study design}

Almost all of the 128 studies had a Control-Exposure (for questions Q2, Q4, Q5) or a Control-Intervention (for question Q1) design, with only two studies having a Before-After-Intervention design (for question Q1).

\section{Taxa studied}

Of all the 128 studies, most were on mammals (78 studies, Fig. 5a), especially on small mammals (32 studies) and bats (26 studies). Birds were the second most studied class, with 45 studies. Amphibians and reptiles were relatively poorly studied, with seven and five studies respectively.

\section{Exposures}

Most of the 128 studies were about roads (69 studies, Fig. 5b) with a focus on birds and small mammals (Fig. 5c). Waterways were the second most studied LTI (37 studies). We found 15 studies about powerlines which also focused on birds and small mammals (Fig. 5c), and 11 studies about railways. The same pattern was found for the specific question Q2 (role of habitat of LTI verges) that concentrated most of the studies (Fig. 5d).

\section{Interventions}

We found eight types of interventions for the specific question Q1 (influence of verge management on the biodiversity hosted by verges) that gathered 21 studies. These studies provided information on the impact of reducing the vegetative biomass of the verges (e.g. mowing, slashing) (six studies), of channelizing waterways (five studies), of increasing waterway water level (three studies), of road lighting (three studies), of using synthetic bottom to construct highway stormwater retention ponds (one study), of allowing humans to come on waterways verges (one study), of improving species habitat on verges (one study), and of planting fleshly-fruited woody species on highway verges (one study). These interventions were thus very diverse with some specific to one LTI (e.g. channelization). 


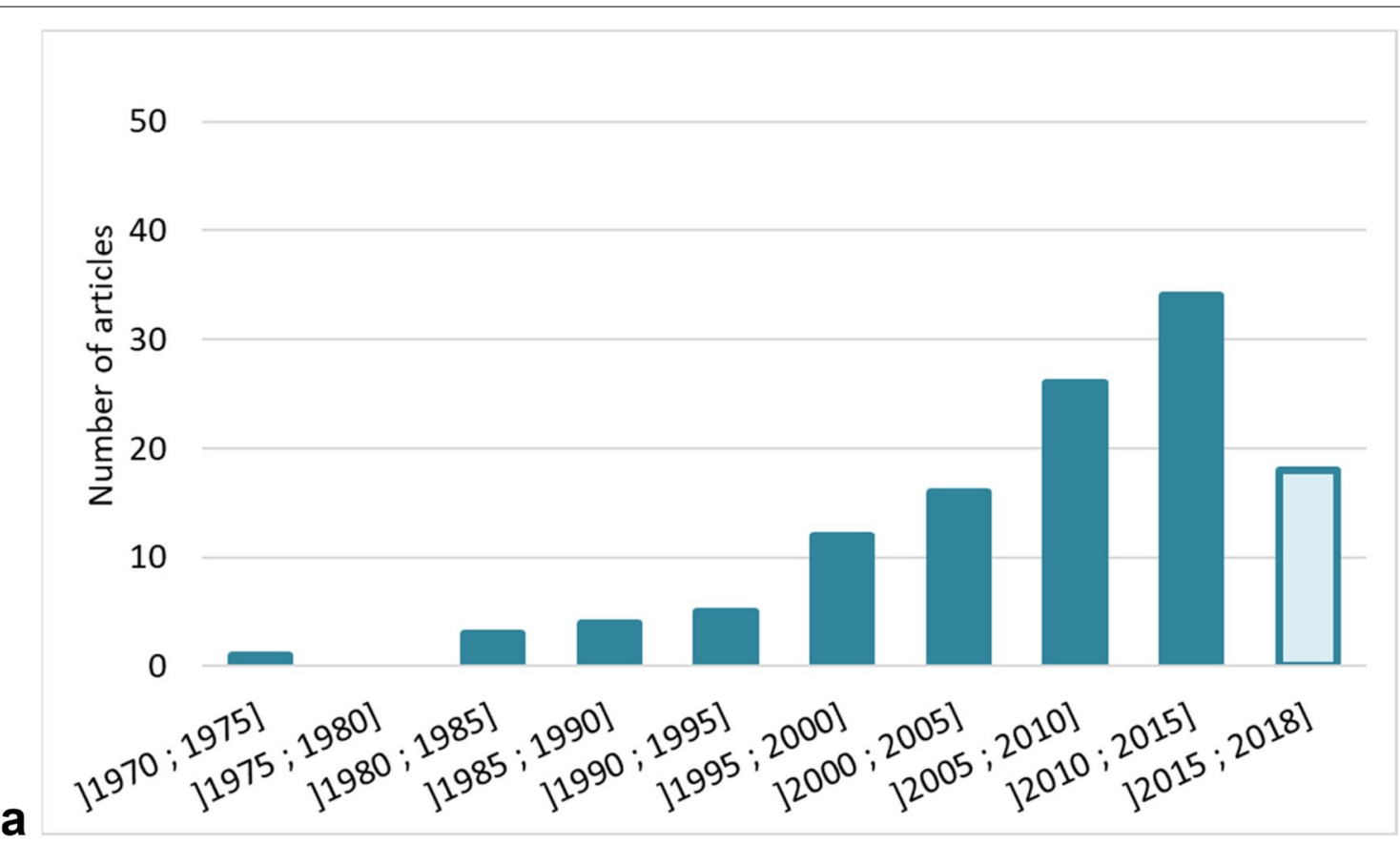

a

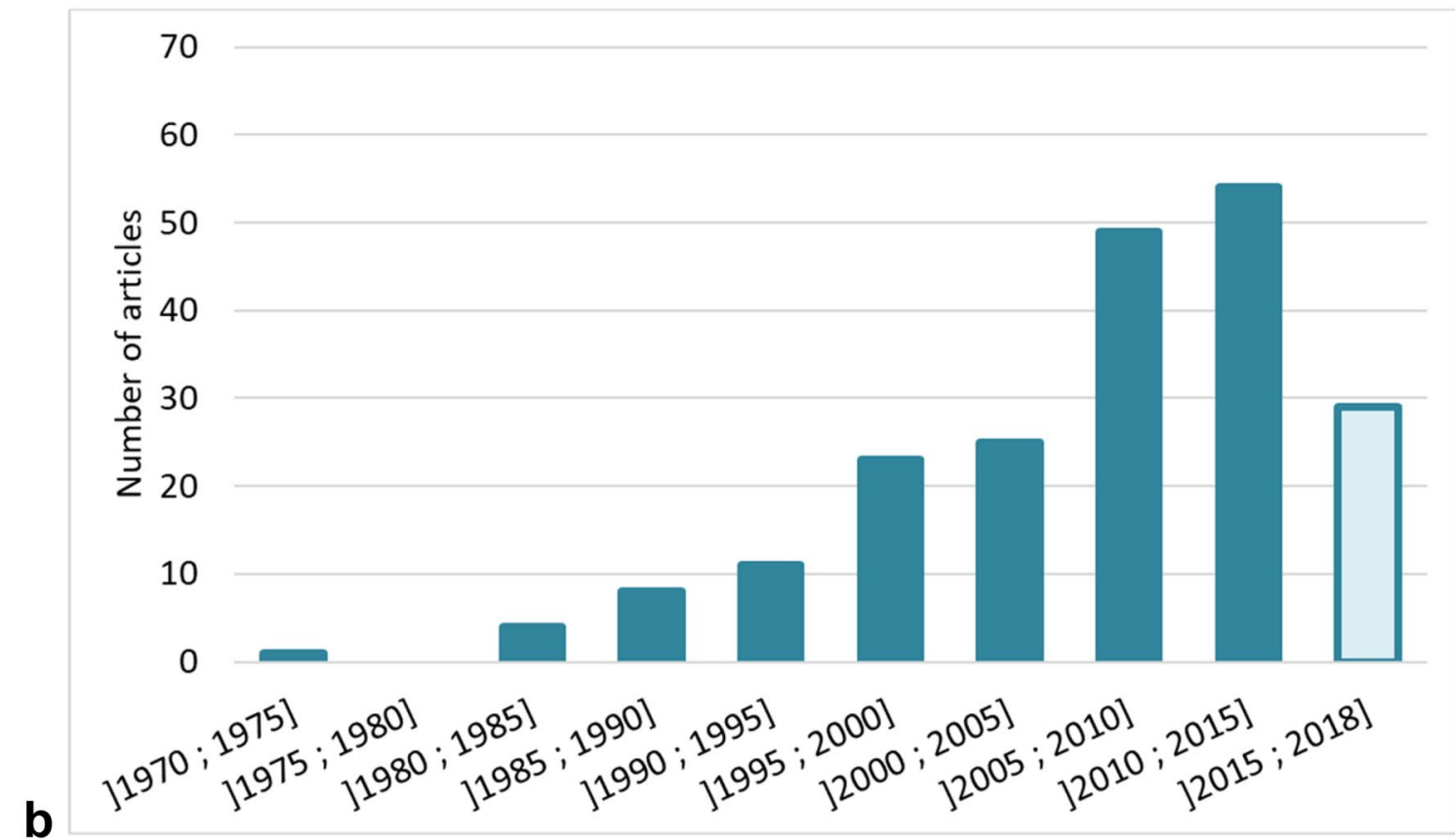

Fig. 3 Years of publication of $\mathbf{a}$ the 119 articles retained after critical appraisal and $\mathbf{b}$ the 204 articles addressing our primary question (before critical appraisal)

\section{Measured outcomes}

Almost $90 \%$ of the studies (113 studies) used measures related to abundance (abundance, density, occurrence, location, activity, ...). Just over a quarter of the studies (33 studies) presented measures of species diversity (mainly species richness but also Shannon index). We found seven studies that provided demographic parameters (survival, reproduction) and six that provided measures 


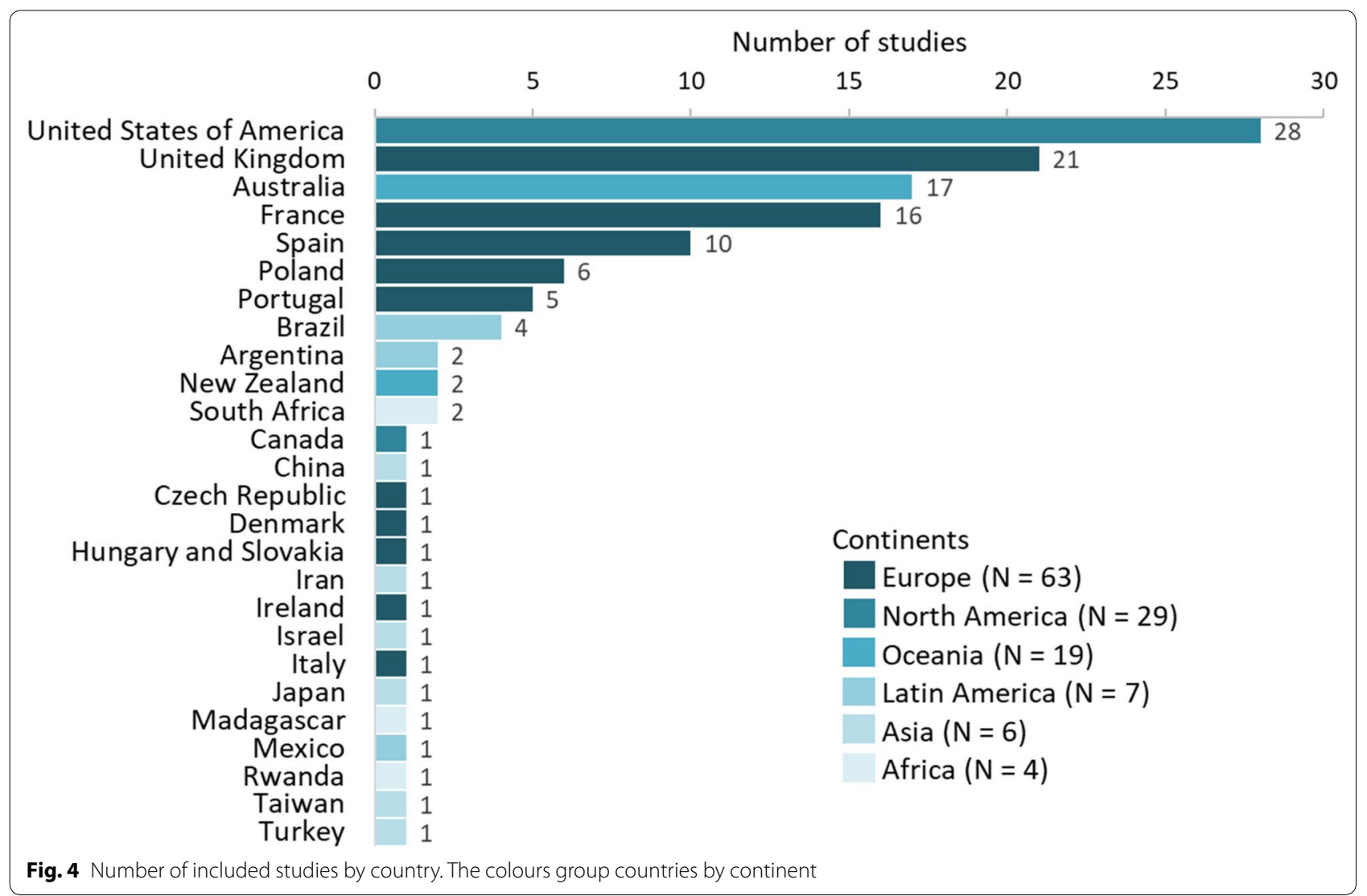

related to similarity between communities (Jaccard similarity coefficient, Bray-Curtis distances) or between populations (genetic distances).

\section{Narrative syntheses}

The narrative syntheses of our six specific questions were based on 91 studies out of the 128 studies with low or medium risk of bias. We set aside the studies that did not provide statistical results to assess the effect of exposure/ intervention (indicated in Additional file 7). The narrative tables summarizing the key results are provided in Additional file 8 .

\section{Is vertebrate biodiversity in LTI verges equal to, higher, or lower than in similar habitats away from LTIs? (question Q2)}

We found 56 studies with statistical results to answer this specific question. Overall, they indicated that vertebrate biodiversity in LTI verges is not found to be statistically different from vertebrate biodiversity in similar habitats away from the LTI, with exceptions for some biological groups and/or LTI. The key results of these 56 studies are summarized in Additional file 8: Table S1. Among them,
- 33 studies (including nine with a low risk of bias) compared vertebrate biodiversity in road verges to similar habitat away from roads (group a in Additional file 8: Table S1);

- 13 studies (including two with a low risk of bias) compared vertebrate biodiversity in waterway verges to similar habitat away from waterways (group b);

- 7 studies (including four with a low risk of bias) compared vertebrate biodiversity in powerline corridors to similar habitat away from powerline corridors (group c);

- 4 studies (all with a medium risk of bias) compared vertebrate biodiversity in railway verges to similar habitat away from railways (group d).

The main results for each LTI and biological group and according to risk of bias are presented in Table 7 . In the following description abundance refers to all outcomes related to abundance (density, occurrence, activity, ...). (Note that a quantitative synthesis (meta-analyses) was also performed on this question $\mathrm{Q} 2$, see further).

Roads Among the nine studies with a low risk of bias, four were about birds [33, 48-50], five about small mam- 

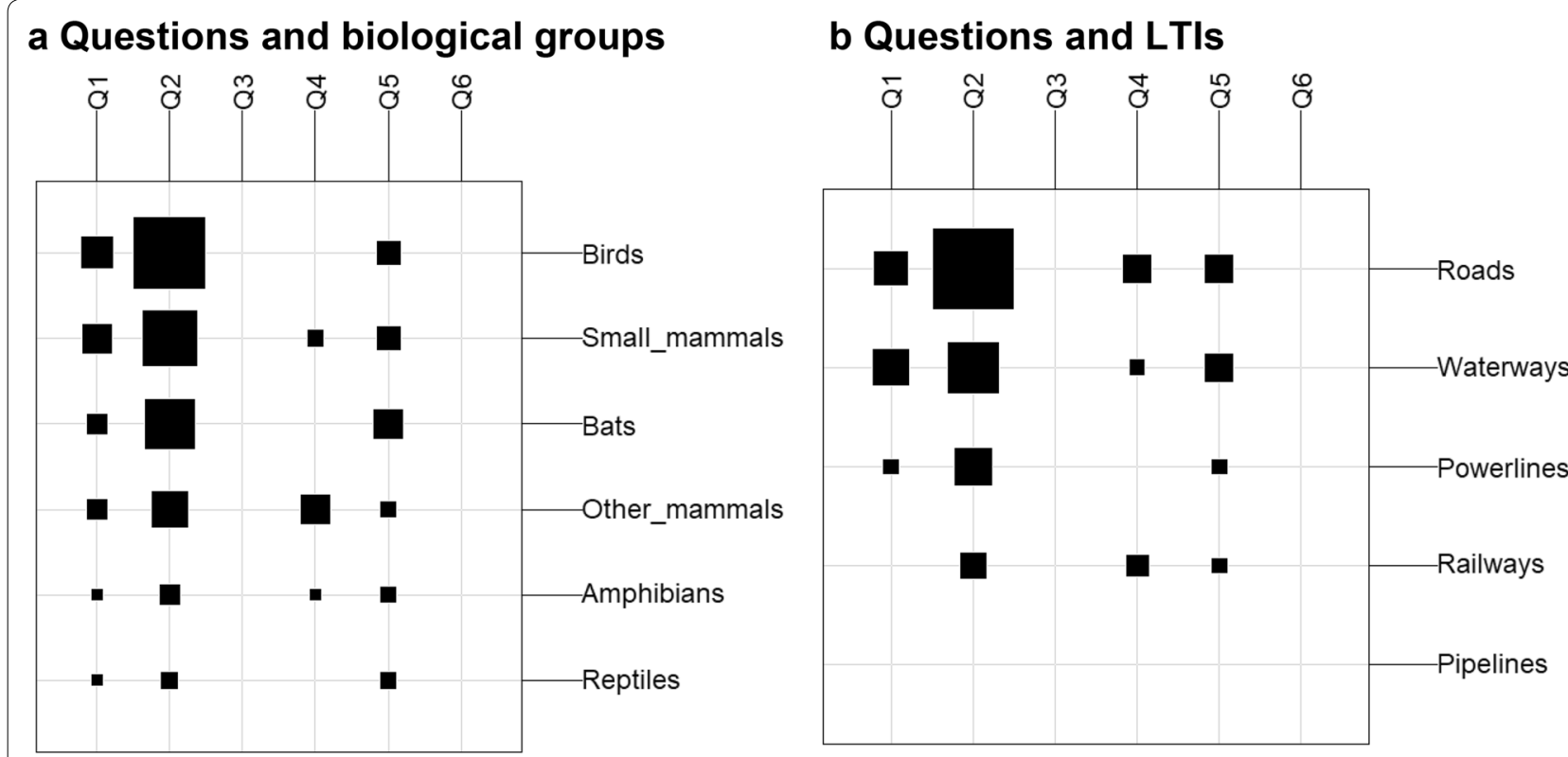

c LTIs and biological groups

\section{d LTIs and biological groups for Q2}
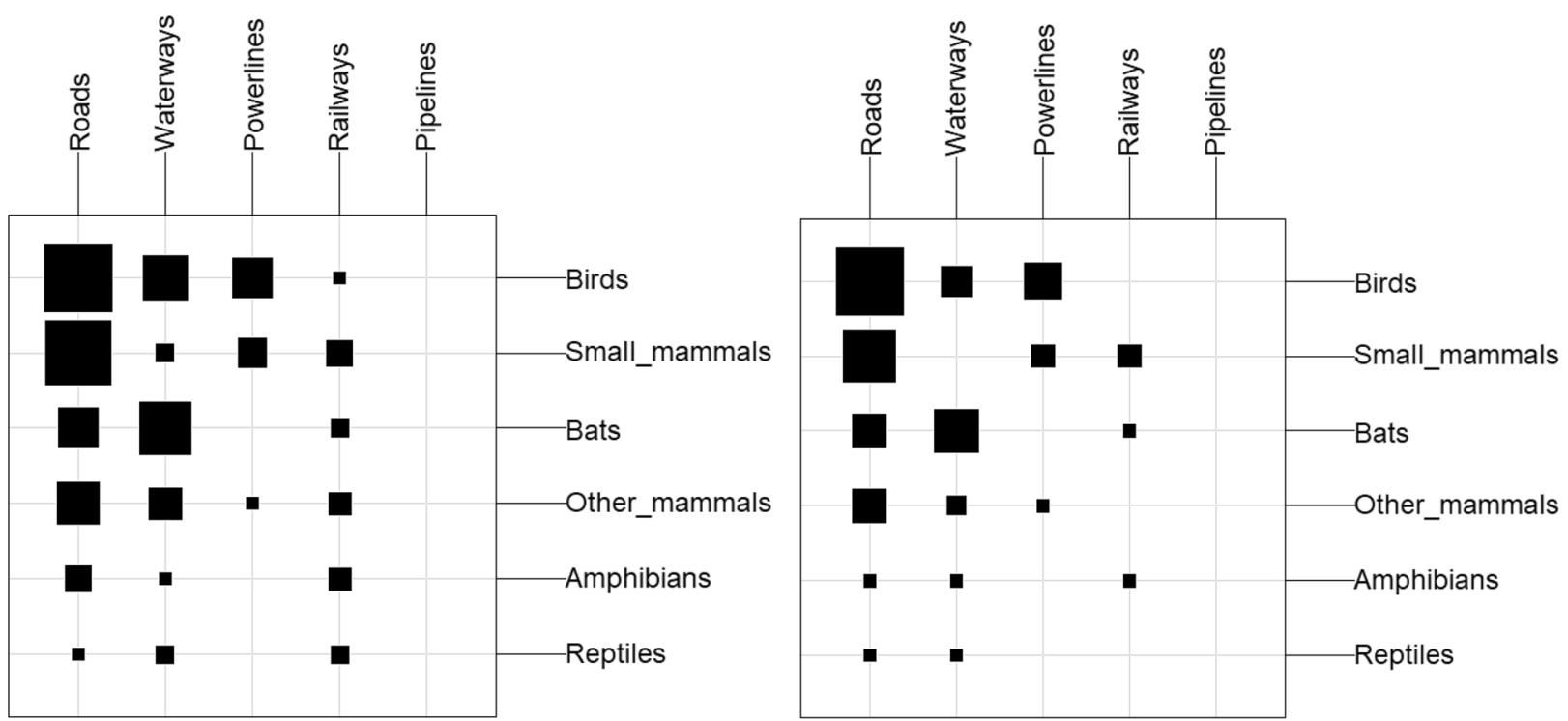

Fig. 5 Distribution of the number of included studies in the six specific questions (from Q1 to Q6, see Table 1), five types of LTIs (roads, waterways, powerlines, railways, and pipelines), and six biological groups (birds, small mammals, bats, other mammals, amphibians, and reptiles). Square size indicates the proportion of studies in each combination of a questions and biological groups, b questions and LTIs, $\mathbf{c}$ LTIs and biological groups, and d LTIs and biological groups for the specific question Q2 (role of habitat of verges)

mals [29, 50-53], and one about avian nest predators [54] from which we could hardly extract evidence as no information was available on species identity.

Studies about birds and small mammals revealed a contrasted pattern between the two groups (Table 7 ,
Additional file 8: Table S1 group a). Studies with a low risk of bias revealed that for birds, species richness [33] or abundance $[33,48]$ was lower in road verges than away from roads, or abundance was not found to be statistically different $[48,49]$ (but there is one exception [48]). 
Table 7 Summary of the main effects found for the specific question Q2 (role of habitat of LTI verges) for each biological group (birds, small mammals, bats, other mammals, amphibians, and reptiles), LTI (roads, waterways, powerlines, and railways), and risk of bias (low or medium)

\begin{tabular}{|c|c|c|c|c|c|}
\hline & Risk of bias & Roads & Waterways & Powerlines & Railways \\
\hline \multirow[t]{4}{*}{ Birds } & Low & (3) & $(1)$ & (3) & / \\
\hline & & $\begin{array}{l}- \text { or NS } \\
{[+ \text { Molothrus ater }]}\end{array}$ & NS or + & $\begin{array}{l}\text { NS or }+ \\
{[- \text { forest interior species }]}\end{array}$ & \\
\hline & Medium & & (2) & $(2)$ & / \\
\hline & & Depends on species & + & $\begin{array}{l}\text { NS } \\
{[- \text { Iridoprocne bicolor }]}\end{array}$ & \\
\hline \multirow[t]{4}{*}{ Small mammals } & Low & (4) & / & $(1)$ & / \\
\hline & & $\begin{array}{l}\text { NS or }+ \\
{[-]}\end{array}$ & & $\begin{array}{l}\text { NS or }+ \\
{[- \text { Antechinus agilis }]}\end{array}$ & \\
\hline & Medium & (5) & / & / & (3) \\
\hline & & NS or + & & & $\begin{array}{l}\text { NS } \\
{[+]}\end{array}$ \\
\hline \multirow[t]{3}{*}{ Bats } & Low & / & / & / & / \\
\hline & Medium & & (8) & / & (1) \\
\hline & & Depends on species & NS or + & & - or NS \\
\hline \multirow[t]{3}{*}{ Other mammals } & Low & / & / & / & / \\
\hline & Medium & (2) & / & $(1)$ & / \\
\hline & & NS or + & & NS or + & \\
\hline \multirow[t]{3}{*}{ Amphibians } & Low & / & / & / & / \\
\hline & Medium & / & (1) & / & / \\
\hline & & & $\begin{array}{l}\text { NS or }+ \\
{[-]}\end{array}$ & & \\
\hline \multirow[t]{3}{*}{ Reptiles } & Low & / & (1) & / & / \\
\hline & & & NS or + & & \\
\hline & Medium & / & / & / & / \\
\hline
\end{tabular}

No study about pipelines were included in the review. All outcomes related to abundance, diversity or demography were considered here but outcomes related to similarity between communities were not. The number of studies is indicated in parenthesis, "- " indicates a negative effect of exposure to LTI (i.e. lower biodiversity in LTI verges compared to similar habitats away from LTIs), "NS" statistically non-significant $(P>0.05)$ differences between LTI verges and similar habitats away from LTI, and "+" a positive effect of exposure to LTI (i.e. higher biodiversity in LTI verges compared to similar habitats away from LTIs). Effects indicated in brackets were exceptions to the main effects reported. Studies with no information on biological group [54] or with results mixed for several groups [76] were not included

For small mammals, species richness [52] or composition $[50,52]$ was not found to be statistically different between road verges and away from road, and abundance was higher $[29,51,52]$ or not statistically different $[29$, $51,53]$. When we considered the studies with a medium risk of bias, it overall revealed the same patterns found for small mammals [32, 55-58] and birds [59-64]. For birds, four studies however reported higher abundance or reproductive success in road verges than away from roads for Dendroica cerulea [65], Pica pica [66], Tyto furcata [67], and Corvus corax [68].

In addition, we found six studies providing information on bat biodiversity in road verges, all with a medium risk of bias. They showed that bat diversity was higher [69] or not found to be statistically different [70] along roads than away from roads. For abundance they reported contrasted results depending on species [69-74].

We additionally found two studies with a medium risk of bias providing information on other mammal biodiversity in road verges. One on raccoons Procyon lotor in the United States of America showed that they selected more road habitat than open habitat [75]. And a study on swamp wallabies Wallabia bicolor in Australia showed that their abundance was not statistically different between close to roads and far from roads [30].

Finally, we found one study with a medium risk of bias conducted in China on the activity of seed predators (mammal and bird species, indistinguishable) that showed that their abundance was higher at roadsides than away from roads in autumn and winter, but not in spring [76].

Waterways Eight studies were about bats [77-84], three about birds [37, 85, 86], one about amphibians [35], and one about reptiles [87]. Overall, they indicated that vertebrate biodiversity in waterway verges was not found to be statistically different or was higher to that away from waterways (Table 7, Additional file 8: Table S1 group b). These studies related to relatively natural waterways, except three related to an artificial managed channel [79], canals [80], and rivers in an urban context [85]. 
Powerlines Four studies presented a low risk of bias, among which three were on birds $[48,88,89]$ and one on small mammals [90]. Overall, they indicated that richness or abundance of bird or small mammal species in powerline verges was not found to be statistically different or was higher than away from powerlines, except for the abundance of forest-interior birds [48] in the United States of America, and the abundance of the small mammal species Antechinus agilis in Australia [90] (Table 7, Additional file 8: Table S1 group c). When we considered the three studies with a medium risk of bias, they overall revealed the same pattern except for the bird species Iridoprocne bicolor, which showed a reduction in breeding success under powerlines compared to away from powerlines [91].

Railways Three studies were about small mammals and were conducted in South America [92-94] and one was about bats and was conducted in France [95]. They were all with a medium risk of bias. Overall, they indicated that biodiversity in railway verges was not found to be statistically different from that away from railways, but more studies are needed to be able to really conclude (Table 7, Additional file 8: Table S1 group d).

\section{Is vertebrate biodiversity in LTI verges dependent on the surrounding landscape? (question Q5)}

We found nine studies with statistical results to answer this specific question. Overall, they indicated that indeed the vertebrate biodiversity of LTI verges depends on the surrounding landscape. The key results of these nine studies are summarized in Additional file 8: Table S2. Among them,

- 5 studies (two with a low risk of bias and three with a medium risk of bias), assessed the effect of the proportion of urbanisation and agriculture or woodland in the landscape (quantitative assessment, group e in Additional file 8: Table S2);

- 4 studies (one with a low risk of bias and three with a medium risk of bias) assessed the effect of the type of landscape (qualitative assessment, group f).

Among the studies that conducted a qualitative assessment, all reported an effect of the type of landscape [51, 64,96 ] or level of altitude [97] on vertebrate (small mammals, birds, bats) abundance or richness.

Among the studies that conducted a quantitative assessment, the two studies with a low risk of bias suggested that urbanisation has a neutral or negative effect on vertebrate biodiversity in LTI verges. These two studies were conducted in Europe on bats and waterways $[98,99]$. Both studies reported neutral effects of the surrounding landscape on bat activity or bat presence (Nyctalus spp., Pipistrellus pipistrellus, P. pygmaeus, Myotis daubentonii and M. capaccinii), except Lintott et al. [98] who reported a negative relationship between the proportion of urbanisation in the surrounding $3 \mathrm{~km}$ and the activity Myotis spp. (activity was more than half reduced when the proportion of urbanisation increased from 10 to $40 \%$ ). The three studies with a medium risk of bias also reported a negative effect of urbanisation on Myotis daubentonii activity along waterways [100], on amphibian species richness in highway verges [101], and on shrubland bird species richness and abundance in powerline right-of-ways [102].

In addition, the two studies with a low risk of bias reported a neutral effect of increasing forest cover in the landscape on bat species activity along waterways [98, 99]. The study of Langton et al. [100], with a medium risk of bias, however reported a positive effect of increasing proportion of woodland on $M$. daubentonii activity along waterways.

Finally, two studies with a medium risk of bias showed that the effect of the proportion of arable or agricultural land in the landscape was neutral on M. daubentonii activity along waterways [100], and negative on shrubland bird species richness and abundance in powerline right-of-ways [102].

\section{Do LTI verge management practices increase, decrease, or have no effect on vertebrate biodiversity in LTI verges? (question Q1)}

We found 19 studies with statistical results to answer this specific question, all of them having a medium risk of bias. Overall, they covered a wide variety of verge management practices that can indeed influence vertebrate biodiversity in verges. Their key results are summarized in Additional file 8: Table S3. Among them,

- 17 studies were about verge management practices implemented for the functioning of the infrastructure (e.g. vegetative biomass reduction, waterway channelization, or road lightening, group g in Additional file 8: Table S3);

- 2 studies were about verge management practices implemented for enhancing biodiversity in verges (group h).

Vegetative biomass management Five studies were about the reduction of verge vegetative biomass, all about small mammal abundance in road verges or powerline corridors. From these studies it emerges that reducing verge vegetative biomass decreases or has no effect on small mammal abundance $[57,103,104]$, except for the intro- 
duced house mouse Mus domesticus that benefits from clearings in powerline corridors in Australia [90, 105]. Interestingly, Meunier et al. [57] evidenced an effect of the type of landscape on the response of small mammals to management: the abundance (number of captures per 60 trap-nights) of the greater white-toothed shrew Crocidura russula was two and a half and three times lower in mown habitat than in unmown habitat in "garrigue" and "plantation" landscapes, respectively, but differences were not found to be statistically significant in the "farmland" landscape. Similarly, abundance of the wood mouse Apodemus sylvaticus was three times lower in mown habitat than in unmown habitat in "plantation" landscape, but differences were not found to be statistically significant in the "garrigue" landscape.

Waterway channelization Four studies focused on waterway channelization, two on river bird species (Riparia riparia, Motacilla cinerea, and Alcedo atthis), one on the European mink Mustela lutreola, and one on the Taiwanese freshwater turtle Mauremys sinensis. The two studies on river bird species found no effect of relatively low levels of waterway channelization on species occurrence [106] or occurrence of breeding territories [107]. The other two studies found a negative effect of relatively high levels of waterway channelization, that decreased European mink occurrence [108] (Jacob index of electivity which is bounded between -1 and 1 fell from 0.37 for natural rivers to -0.78 for aggressively canalised rivers) or turtle Mauremys sinensis overall abundance by $14 \%$ [109]. The negative effect of a high level of channelization on the turtle population was however complex, with a decreasing abundance of females and juveniles but an increasing abundance of males after the intervention.

Increasing waterway water level Three studies assessed the impact of increasing waterway water level on bird species occurrence [110, 111], abundance or diversity [112]. Overall, they reported contrasted results depending on the species and the season considered. However one study assessing variation in Danube river at the border between Hungary and Slovakia reported a strong tendency for species richness and abundance to correlate negatively with water level and for Shannon diversity to correlate positively [112]. A national study across Great Britain similarly reported that the study species presence displayed largely negative association with mean daily flow [111].

Road lighting Three studies assessed the effect of road lighting on bat activity, in United Kingdom or Ireland. The studies reported a negative impact of lighting on Rhinolophus ferrumequinum activity [113], a positive impact on Nyctalus leisleri activity [114], and a neutral impact on Pipistrellus pipistrellus and P. pygmaeus activity [114]. One study reported contrasted effects of lighting on bat (probably P. pipistrellus) activity depending on the colour of the lamps: bat activity along roads lit by white streetlamps was higher than along unlit roads, and activity along roads lit by orange streetlamps was lower than along unlit roads [115].

Other management practices (not designed for biodiversity) Finally, one study assessed the effect on amphibian richness of using a synthetic bottom to construct stormwater retention ponds along highways [101], and reported statistically non-significant effect of using such a synthetic material. Another study assessed the impact of human presence along rivers in Glasgow, Scotland, on waterbird presence [116], and showed contrasted effects (positive, negative, or statistically non-significant effect) depending on the species and the season considered.

Management practices specifically designed for biodiversity Two studies dealt with management practices that were directly implemented for enhancing biodiversity in verges. One study was about improving European otter Lutra lutra habitat in waterway verges through fencing to keep livestock from grazing at the water edge, planting trees and shrubs, reconstructing oxbow, and constructing artificial holts [117]. From 2 to 4 years after the intervention, the authors found a positive effect on otter incidence. The other study was about clump planting fleshly-fruited woody species on highway embankments, to enhance bird seed dispersal on the roadsides, in a context of highly deforested agricultural surrounding landscape [118]. One year after the intervention, the authors did not find any statistically significant difference in species richness nor abundance between embankments with or without plantings.

\section{Are vertebrate movements in LTI verges equal to, higher, or lower than their movements in similar habitats away from LTIs? (question Q4)}

We found seven studies with statistical results to answer this specific question, all of them about mammals. Overall, they indicated that mammal movements on LTI verges are not statistically different or higher than their movements in similar habitats away from the LTIs, suggesting that LTI verges can constitute corridors for mammal displacements. The key results of these seven studies are summarized in Additional file 8: Table S4. Among them,

- 4 studies (two with a low risk of bias and two with a medium risk of bias) conducted direct measures of vertebrate movements with radio-telemetry or cap- 
ture-mark-recapture methods (direct assessment, group i in Additional file 8: Table S4);

- 3 studies (one with a low risk of bias and two with a medium risk of bias) conducted an indirect assessment of vertebrate movements through predation rate or through genetic distance analyses (indirect assessment (group j)).

The two studies with a low risk of bias and direct assessment of mammal movements in LTI verges were conducted in Australia and were about roads. These studies found that Tasmanian devils (Sarcophilus harrisii) and wild dogs (Canis lupus dingo, C. l. familiaris and hybrids of the two) were more likely to move along roads than to move along other linear features such as tracks or watercourses $[119,120]$. For the spotted-tailed quoll (Dasyurus maculatus) no differences were found [119]. The two other studies that assessed mammal movement along LTI verges had a medium risk of bias and found statistically non-significant differences ([121] about roads and [122] about railways). Similarly, all studies that indirectly assessed mammal movements along LTI verges through predation rate of bird eggs [123] (according to the "travel line" hypothesis) or genetic distance analyses [124, 125] reported statistically non-significant differences.

\section{Are vertebrate movements in LTI verges dependent on the surrounding landscape? (question Q6)}

No study evaluated the influence of the surrounding landscape on vertebrate movements in LTI verges.

\section{Do LTI verge management practices increase, decrease, or have no effect on vertebrate movements in LTI verges? (question Q3)}

No study evaluated the influence of verge management on vertebrate movement in LTI verges.

\section{Data synthesis: quantitative syntheses Description of the data}

We extracted quantitative data from 35 studies among the 82 with low or medium risk of bias addressing the specific question Q2 (Is biodiversity in LTI verges equal to, higher, or lower than in similar habitats away from LTIs?). Among the 136 cases extracted from these 35 studies, 99 cases in 31 studies referred to species or group abundance, and 37 cases in 14 studies referred to species richness (Additional file 9). We estimated the variance of cases with data imputation (i.e. filling missing variance by using the available data from the other studies) for two cases $(1.5 \%$ of the total data). When several cases were extracted from the same study, this was because there was data for several species or group of species (eight studies), for several sites (eight studies) or for several years or seasons (three studies).

Most of the cases in our data were conducted along roads (highways $40 \%$ and non-highway roads $26 \%$ ), then along waterways $(18 \%)$, powerlines $(10 \%)$, and railways (6\%). No study was conducted along pipelines. Cases were mostly from Europe [53\%, especially from France (41\%) and Poland (8\%)], then from North America (26\%, all in the United States of America) and Oceania (12\%). South America, Africa, and Asia represented 4, 3, and 1\% of the data, respectively.

The majority of the cases were about birds (54\%), then bats (21\%) and small mammals (19\%). The other biological groups were represented by only six cases in a unique study in Australia on the swamp wallaby Wallabia bicolor (other mammals), one case also in Australia on the scincid lizard Menetia greyii (reptiles), and one case in Brazil on terrestrial amphibians.

\section{Meta-analyses and publication bias}

For abundance, we found that, overall, vertebrates were more abundant along verges than in similar habitats away from LTIs (grand mean effect size $d=0.51,95 \%$ confidence interval $(95 \% \mathrm{CI})[0.09 ; 0.94])$. We also found a statistically significant heterogeneity in the effect sizes $\left(Q_{T}=1286.94\right.$, $\mathrm{P}$-value $\left.<0.0001, \mathrm{~N}=99\right)$, indicating that moderators could explain variations in effect sizes.

For species richness, the overall grand mean effect size was not statistically different from zero $(d=-0.15,95 \%$ CI $[-1.08 ; 0.78])$. The negative value of the effect size mainly came from a highly influential case (Cook's distance above 0.5 , see after). When this case was removed, the grand mean effect size became positive but still not statistically different from zero $(\mathrm{d}=0.19,95 \% \mathrm{CI}[-0.57$; $0.94]$ ), indicating that species richness in LTI verges was overall similar to species richness in control sites away from LTIs. We found a statistically significant heterogeneity in the effect sizes $\left[Q_{T}=762.73\right.$, P-value $<0.0001$, $\mathrm{N}=36$ (without the influential case)], indicating that moderators could explain variations in effect sizes.

We did not find obvious publication bias when we examined the funnel plots and the plot of the cumulative meta-analysis by publication year, neither for abundance nor for species richness (Additional file 10). Egger's regression test for funnel plot asymmetry indicated that there was no statistically significant asymmetry for abundance $(\mathrm{P}$-value $=0.80)$. For species richness, asymmetry was statistically significant $(\mathrm{P}$-value $<0.0001)$ but when we removed from the data the highly influential case, funnel plot asymmetry was no longer statistically significant (P-value $=0.63$, Additional file 10). Effect sizes were also not correlated to publication year, neither for 
abundance (Pearson's correlation coefficient $r=-0.04$, $\mathrm{P}$-value $=0.68, \mathrm{~N}=99)$ nor for species richness $(r=0.18$, $\mathrm{P}$-value $=0.30, \mathrm{~N}=36$, without the influential case). For abundance, the Rosenberg fail safe number was 6527, which is far greater than $5 k+10(5 * 99+10=505)$, suggesting the estimated positive grand mean effect size was robust against potential publication bias. For species richness, the grand mean effect size was not statistically significantly different from zero thus we did not compute the Rosenberg fail safe number. Finally, we did not detect any influence of the risk of bias of the study (low or medium) on effect sizes (after removing the effect of type of LTI) neither for abundance $\left(Q_{M}=1.86\right.$, P-value $\left.=0.39, \mathrm{~N}=99\right)$ nor for species richness $\left(Q_{M}=0.08, \mathrm{P}\right.$-value $\left.=0.96, \mathrm{~N}=37\right)$.

\section{Effects of moderators on abundance}

For abundance, we analysed the effect of biological groups on vertebrate response to each LTI separately (i.e. we analysed five subsets of the data, Fig. 6). We discarded the reptile group as only one case was available (Additional file 9), as well as pipelines as we did not find any case corresponding to this LTI.

We found differences in the response to highways according to biological groups $\left(Q_{M}=12.77\right.$, $\mathrm{P}$-value $=0.005, \mathrm{~N}=37$ ). Birds were less abundant in highway verges than in control sites contrary to small mammals that were more abundant in highway verges than in control sites (Figs. 6 and 7). For the category "other mammals", which is actually one study with six cases about swamp wallaby Wallabia bicolor in Australia, their abundance in highway verges was not found to be statistically different from their abundance in control sites.

We also found differences in the response to waterways according to biological groups $\left(Q_{M}=9.51\right.$, $\mathrm{P}$-value $=0.009, \mathrm{~N}=16)$. Birds were more abundant in waterway verges than in control sites (Figs. 6 and 7). We found the same pattern for bats but it was not statistically significant (P-value $=0.195$, Fig. 6).

For non-highway roads $(\mathrm{N}=26$, birds, small mammals, and bats), powerlines $(\mathrm{N}=11$, birds and small mammals), and railways $(\mathrm{N}=8$, small mammals and bats), we did not find any statistically significant effect of biological groups on the response to LTIs (Figs. 6 and 7). For these groups in these LTIs, abundance was not found to be statistically different between verges and control sites.

We ran the analyses on different subsets of data analysing the effect of the type of LTI on bird, small mammal, and bat response to LTIs separately (i.e. analysing three subsets of data, Fig. 8). We found similar results to the previous ones, except that the negative pattern for bird abundance response to highways was not statistically significant $(P$-value $=0.098)$, and that the positive pattern for bat abundance response to waterways became statistically significant $(P$-value $=0.002)$.

\section{Effects of moderators on species richness}

For species richness, we analysed the effect of the type of LTI on bird response to LTIs (Fig. 6). We only considered birds as only three cases were available on bats, one on small mammals, and one on amphibians (Additional file 9). Moreover, we did not consider powerlines as the analysis of Cook's distance revealed a highly influential case ("Kroodsma_1987_2", Additional file 9) that was removed, leaving only one case. Pipelines and railways were also discarded as there were no available cases.

We found differences in the response of bird species richness to LTI according to the type of LTIs $\left(Q_{M}=24.85\right.$, P-value $<0.0001, N=30$ ). Similarly as for abundance, bird species richness was lower in highway verges than in control sites (Fig. 9). At the opposite, bird species richness in waterway verges was higher than in control sites. For non-highway roads, species richness in verges was not found to be statistically different from that in the control sites.

\section{Discussion}

In this paper, we searched for evidence on the role of habitat and/or corridor for vertebrates (excluding fishes) of linear transportation infrastructure (LTI) verges in temperate ecosystems. We found 128 studies with low or medium risk of bias answering this broad question, most of them specifically addressing the role of habitat of LTI verges $(64 \%)$ and the influence of management practice $(16 \%)$ and surrounding landscape (13\%) on this role of habitat. Studies were mainly conducted in Europe and in the United States of America (71\%), and largely focused on road verges $(54 \%)$. The taxa studied were mainly birds (35\%), small mammals (25\%) and bats (20\%). The number of studies on the question was found to increase exponentially over time, suggesting that more evidence may complete our findings in the coming years. Our results revealed that the potential of linear transportation infrastructures verges to constitute a habitat for vertebrate species varies according to the infrastructure and the biological group considered. Relatively natural waterways could be positive for the abundance of birds and bats. Highways can benefit to small mammals, that generally respond positively to human disturbance, but seem detrimental to passerine birds. The amount of literature gathered for powerlines (11 studies) and railways (eight studies) prevent us to draw clear conclusions for these LTIs. 


\section{ABUNDANCE}

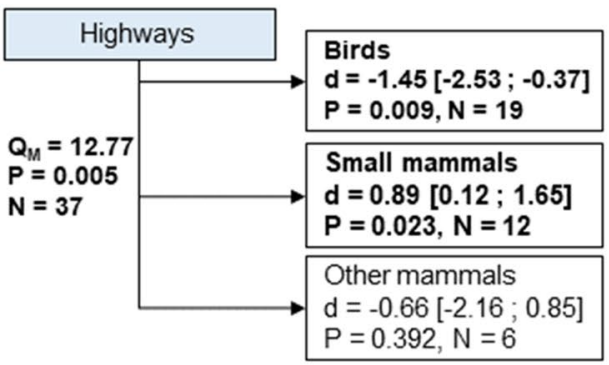

\section{SPECIES RICHNESS}

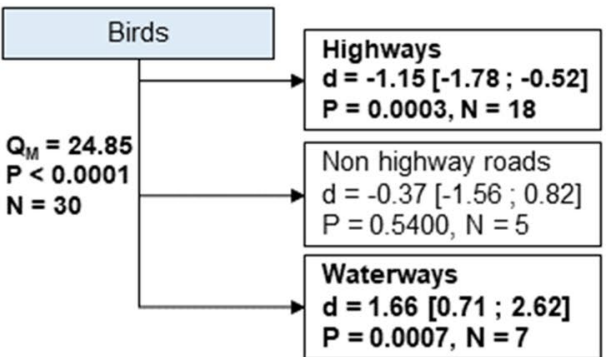

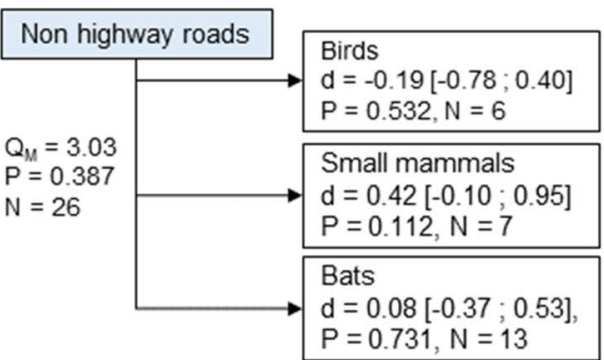
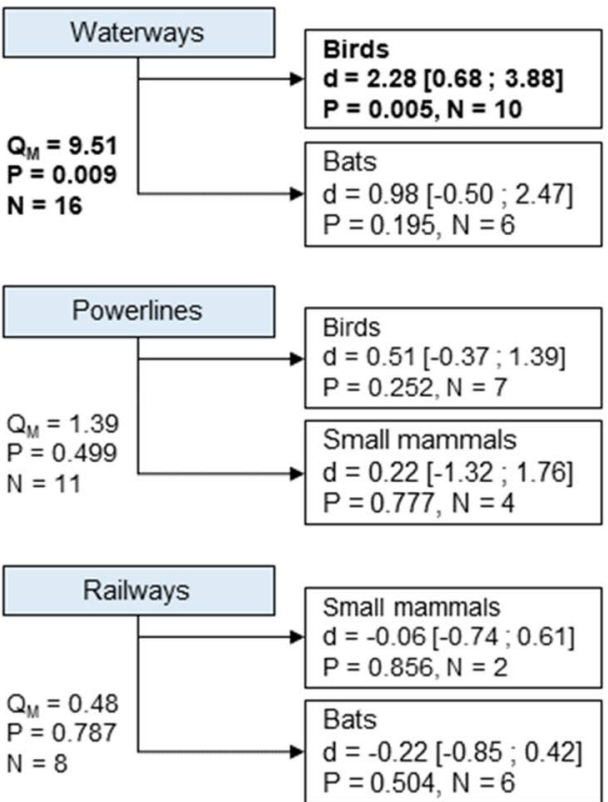

Fig. 6 Summary flow chart of the meta-analyses and results addressing our specific question Q2 (Is biodiversity in LTI verges equal to, higher, or lower than in similar habitats away from LTIs?). For abundance, data were analysed for each LTI separately (five subsets of data), and for species richness data were analysed for birds only. The statistical results presented are $Q_{M}$ the between-class heterogeneity, $P$ the $P$-value, $N$ the number of cases, and d the estimated mean effect size with 95\% confidence intervals in brackets. Statistical significance (P-value < 0.05$)$ is highlighted in bold

\section{Reasons for heterogeneity}

\section{Highway verges may be a refuge for small mammals} but seem detrimental to passerines

Studies providing information on the role of road verges as habitats (question Q2) revealed contrasted patterns between birds (mostly passerines) and small mammals.
Small mammal abundance was higher in highway verges than away from highways and bird species richness and abundance was lower in highway verges than away from highways (Figs. 7 and 9, we did not analyse the response of small mammal species richness with a meta-analysis because of the lack of available cases). 


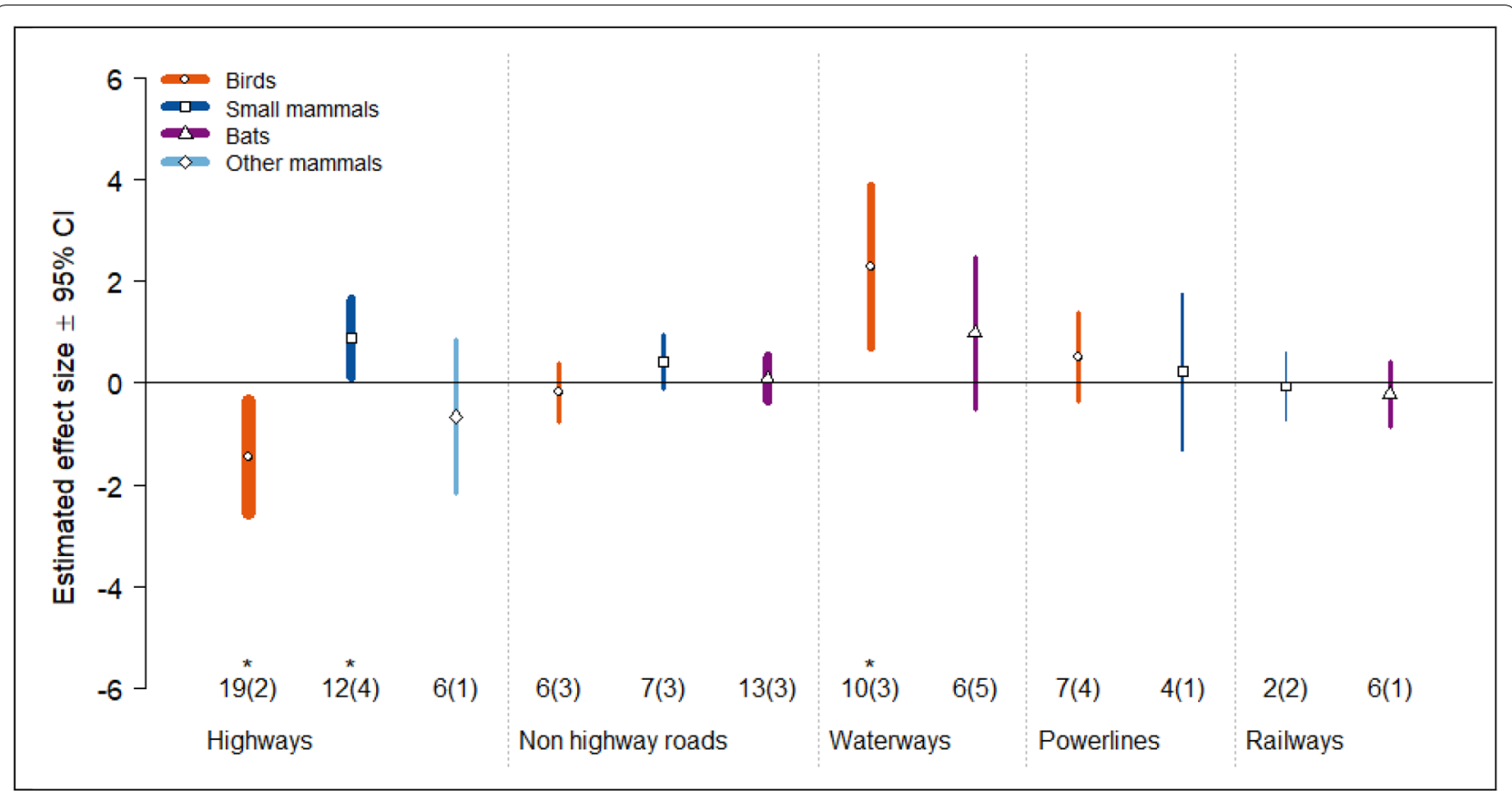

Fig. 7 Estimated mean effect sizes of the comparison of abundance between LTI verges and similar habitats away from LTIs, by LTI and biological group, with $95 \%$ confidence intervals. A positive effect size indicates that the abundance is higher in verges than in control sites. The thickness of the bars is proportional to the number of cases, which is indicated below each estimated mean with the corresponding number of studies in brackets. Estimated means statistically significantly different from zero (P-value $<0.05)$ are indicated by a star (*) $^{*}$

The higher abundance of small mammals in highway verges may be first explained by their ecological strategy, as small body mass, short generation length, high litter/clutch size, habitat generalist and invertivorous mammal species are predicted to be positively affected by human disturbance [126]. The higher abundance of small mammals in highway verges may also be explained by their behaviour of avoiding the road surface combined with a release from predation when living in verges [19, 127, 128]. Species that avoid going onto roads and have small movement ranges, small territory sizes and high reproductive rates may be weakly affected by roads because viable populations are likely to persist within areas bounded by roads. Moreover, if these small species are prey for larger species that are negatively affected by roads, their abundance may actually be indirectly positively related to roads, due to the release from predation in roadside areas. It was indeed shown that some small mammals avoid going onto roads $[129,130]$ and that several predators of small mammals are negatively affected by roads, including foxes, badgers, and snakes [19]. However, the predation release hypothesis for positive road effects on small mammals has to be clearly demonstrated [131], although the hypothesis was again supported by a recent study showing that predation rate on artificial nests was reduced up to $25 \mathrm{~m}$ from a highway with high traffic density [132] (but see [133]). The release from predation in roadside areas may only occur on roads with high traffic density such as highways. We indeed did not find higher abundance of small mammals in non-highway verges than away from verges (abundance was not statistically different).

This result of a higher abundance of small mammal in highway verges should not be generalized and may depend on the quality of the surrounding habitat (e.g. influence of landscape) or on the quality of road verges (e.g. influence of management) within each habitat [134]. For instance, Ascensao et al. [51] found an effect of the type of landscape on the response of the wood mouse Apodemus sylvaticus to highways (but not of the western Mediterranean mouse Mus spretus or the greater whitetoothed shrew Crocidura russula), and highlighted the importance of vegetation cover on verges. Also, Meunier et al. [57] showed that abundances of $A$. sylvaticus and $C$. russula differed in mown vs. unmown highway verges in three different landscapes ("garrigue", "plantation", "farmland"). They further explained that, in the farmland landscape, highway verges, like other habitats that are stable in time, seem to act as a refuge for many small-mammal species inhabiting crops during the period of intensive harvesting. 

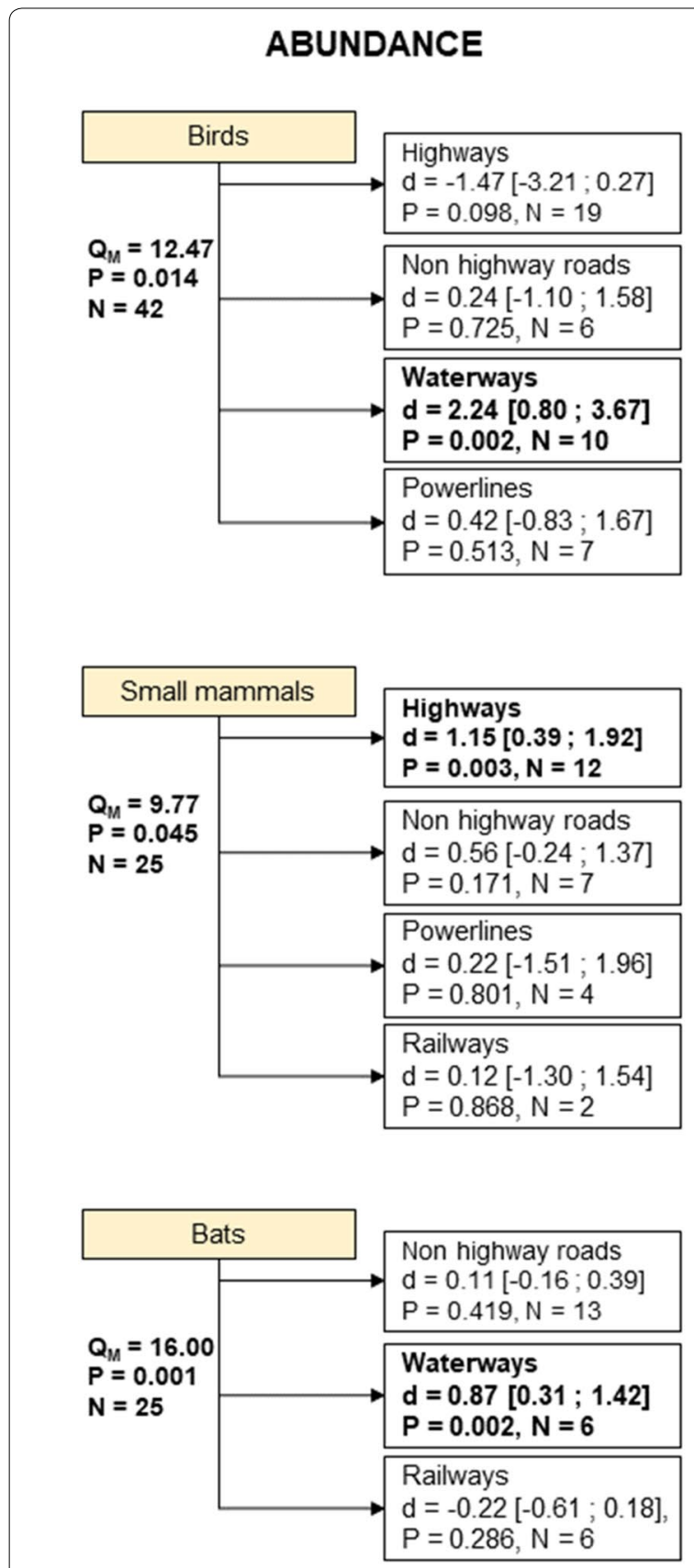

Fig. 8 Summary flow chart of the additional meta-analyses performed on abundance and their results. Instead of splitting the data according to the type of $L T I$, we split the data according to biological groups. The statistical results presented are $Q_{M}$ the between-class heterogeneity, $\mathrm{P}$ the $\mathrm{P}$-value, $\mathrm{N}$ the number of cases, and $d$ the estimated mean effect size with 95\% confidence interval in brackets. Statistical significance $(P$-value $<0.05)$ is highlighted in bold

In contrast to small mammals, we found a lower abundance (and species richness) of birds-mostly passerines-in highway verges than away from highways. This result may be explained by both their high vulnerability

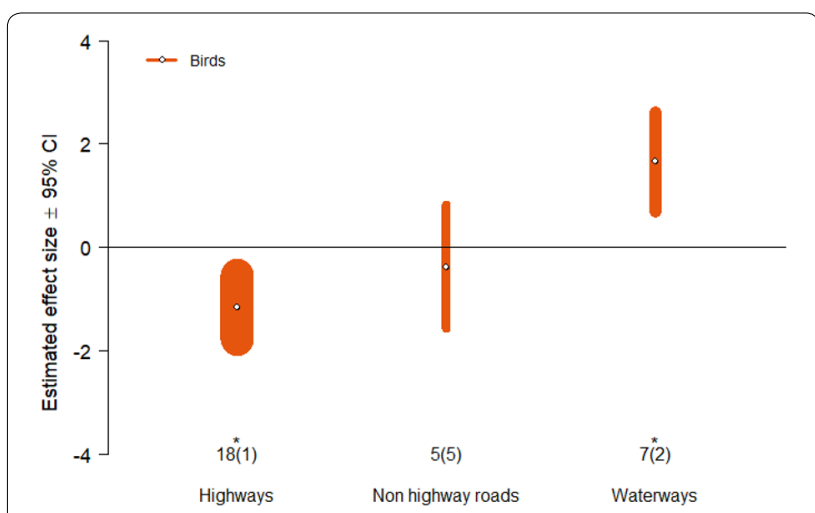

Fig. 9 Estimated mean effect sizes of the comparison of bird species richness between LTI verges and similar habitats away from LTIs, by LTI, with 95\% confidence intervals. A positive effect size indicates that the species richness is higher in verges than in control sites. The thickness of the bars is proportional to the number of cases, which is indicated below each estimated mean with the corresponding number of studies in brackets. Estimated means statistically significantly different from zero ( $P$-value $<0.05$ ) are indicated by a star $(*)$

to traffic noise disturbance and their high vulnerability to road mortality $[19,135]$. First, it has been shown that noise disturbance due to traffic reduces bird abundance and species richness along roads $[136,137]$ (but noise might induce a bias in bird detectability). Noise disturbance can mask the acoustic signals used by birds for communication, cause physiological stress, changes in vocal communication or foraging behaviour, with negative consequences on survival and reproductive success or causing avoidance of the noisy area [138] (e.g. [139142]). Even for a species that is common in urban areas such as the great tit Parus major, motorway traffic noise was found to have a negative effect on its reproductive success [143]. The negative effect of traffic disturbance through acoustic masking may also vary according to species vocal features. For instance, it was found that birds having lower-pitched vocalisations are more sensitive to noise pollution [144]. Second, the lower abundance and species richness in highway verges than away from highways may be explained by road mortality resulting from collisions with moving vehicles. A high number of birds killed on roads was previously reported in Europe (350,000 to 27 million birds per year depending on the country, [145]) and in the United States of America (more than 80 million per year, [146]). In south-western France, the most frequent bird carcasses encountered along motorways were Passeriformes and Strigiformes [147]. Foraging behaviour and preferred habitat were identified as important characteristics explaining the risk of road mortality for passerines, with small woodland passerines that often forage in shrubs and small trees being the most 
vulnerable to vehicle collision [148]. Thus in rural landscapes, roadside vegetation may attract birds for breeding, foraging, or resting, and act as an ecological trap especially when traffic volume is high [149]. Finally, the negative effect of highways on bird abundance and species richness was no longer statistically significant when looking at non-highway roads. This may be explained by lower traffic on non-highway roads than on highways, causing lower noise disturbance and lower mortality due to collisions with vehicles.

\section{Birds and bats tend to respond positively to waterways}

Studies about the role of habitat of waterway verges were mainly about birds and bats, and revealed a positive response of bird abundance and species richness (Figs. 7, 9) and of bat abundance (Fig. 8) to waterways (we did not analyse the response of bat species richness to waterways with a meta-analysis because no cases were available). The positive effect of waterways on birds and bats may be explained by the relatively natural waterways considered in the studies included in the synthesis, with only three out of the 13 studies concerning artificial managed channels [79], canals [80], or rivers in an urban context [85]. Relatively natural waterways may constitute a feeding habitat for many species and waterways may not only be transport infrastructures but also sources of prey (e.g. for lizards [150] or for spiders [151]). On the one hand, the presence of waterways may benefit to insectivorous birds and bats because of higher insect concentrations along waterways than away from water. It was shown that insectivorous birds are more frequent along a stream in a riparian forest than in upland forest away from the stream, feeding on both insects from the riparian vegetation and aquatic insects emerging from the stream [152]. Besides, a positive correlation between the density of insectivorous birds and emergence production of aquatic insects was observed along streams [153, 154]. Likewise, it was shown that bat activity along a stream is positively influenced by emergence production of aquatic insects, especially in spring when availability in terrestrial insects is low [155]. On the other hand, the presence of high $(>3)$ order streams (considered in our review as navigable waterways) compared to low $(\leq 3)$ order streams may benefit to insectivorous birds and bats because of a higher amount of channel habitat available for the production of emerging insects [154], or because of a higher quality of the riparian vegetation along high order streams [86].

\section{Powerline and railway verges may constitute a suitable habitat for vertebrates}

Overall, the abundance of vertebrates in powerline verges tended to be higher than away from powerlines although the difference was not statistically significant
(Fig. 7). Lower abundance in powerline verges than away from powerlines was however observed for some forestinterior specialist species [48], suggesting that the large opening performed in intact forests for powerline installation may deeply modify species composition through the simultaneous negative impact on forest-interior specialist species but the creation of new habitat for species that prefer open areas [4].

Along railways, the abundance of small mammals and bats tended to be lower than away from railways although the difference was not statistically significant (Fig. 7). More studies are needed to draw clear conclusions. It was recently found that the abundance of birds was not statistically different between along railways and away from railways in farmland landscape [156], suggesting that railway verges may be a suitable habitat for some vertebrate species. Bird species richness in railway embankments was even found to be higher than away from railways, probably because in agricultural landscapes railway verges are important remnants of diverse vegetation, increasing the number of available niches in the landscape [156] and because the noise disturbance due to trains passing at clear intervals is marginal [157].

\section{Heterogeneity in the use of $L T I$ verges as habitat}

In this paper, we synthesised the evidence on the role of habitat of LTI verges for vertebrates, meaning that we sought evidence of occupancy of LTI verges by vertebrates. In our results, we did not distinguish between species that use LTI verges for a short time, for instance to move or to feed (e.g. bats), and species that spend long-time (e.g. for breeding) or that can fulfil all their lifecycle on LTI verges (e.g. small mammals). The duration of occupancy and/or the activities performed by species on LTI verges (e.g. moving, feeding, breeding, living) may however influence the role of LTI verges as a potential habitat.

\section{Knowledge gaps and clusters}

The studies included in our review disproportionately addressed our specific question Q2 (role of habitat of verges), with roads being the most studied infrastructures. Thus for five of our six specific questions, few evidence was found. The knowledge cluster towards roads however hides a knowledge gap for some biological groups. Indeed, the potential of road verges as habitat for amphibians (the most threatened vertebrate group, [158]) or reptiles has been rarely studied (only one and two studies for amphibians and reptiles, respectively). For these two specific groups, the available literature mainly focused on habitat loss and fragmentation, road mortality and mitigation measures (e.g. $[159,160])$. 
Although LTI managers have most interest for the specific questions related to the potential role of corridor of verges, we identified a major knowledge gap with only seven studies included in the review for question Q4 (role of corridor) and no study included for questions Q3 (influence of management on the role of corridor) and Q6 (influence of landscape on the role of corridor). This knowledge gap may first be explained by the technical constraints in effectively measuring the linear movement of vertebrates in LTI verges. Indeed, among the seven studies answering question Q4, only four studies directly assessed movement through radio-telemetry or capturemark-recapture methods, the others conducting an indirect assessment of movements. Second, the low number of included studies for question Q4 may be explained by our inclusion criteria. Indeed, to be included a study had to compare the movement in LTI verges to the movement in similar (i.e. linear) features (comparator). We consequently excluded studies assessing the role of corridor of verges without this appropriate comparator [161-165]. These studies can nevertheless provide information on the role of corridor of verges. For instance, the analysis of genetic distances between Microtus duodecimcostatus and Mus spretus (small mammals) populations in highway verges in Spain indicated no corridor effect of verges [163]. In contrast, the analysis of genetic distances between Muscardinus avellanarius (small mammal) populations in highway verges in Germany indicated that roadside shrubs can provide suitable corridors for dispersal [162]. A connectivity analysis of $M$. avellanarius populations in Germany similarly indicated that roadside hedges and copse can provide suitable corridors [161]. A capture-mark-recapture study of two small mammal species Apodemus flavicollis and Clethrionomys glareolus in highway verges in Germany also revealed longitudinal movements of individuals in verges [164]. Finally, a translocation study of gopher tortoises (Gopherus polyphemus) in the United States of America showed that, instead of being used for movements, roadsides actually acted as residential locations for tortoises to remain sedentary for weeks at a time [165].

Finally, pipelines were clearly understudied as no study about pipelines were included in our review. The lack of knowledge on the impact of pipelines may concern all biological groups and not only vertebrates. For instance in the systematic review on insect biodiversity in LTI verges, only one study about pipelines was included [22]. One reason that may explain this lack of knowledge is that buried pipelines are thought to have limited negative impact on wildlife compared to other LTIs such as roads. However, impacts of pipelines on biodiversity are often complex and difficult to predict [166], thus this lack of knowledge may be problematic in a context of increasing demand of energy throughout the world.

\section{Review limitations}

First, some biases in our review may arise from several decisions taken during the review process. On one hand, potential biases may arise because, due to time constraints, we excluded the articles that did not provide any abstract $(\mathrm{N}=1235$, Additional file 11$)$, which is not consistent with the guidelines of the Collaboration of Environmental Evidence, which recommend directly allocating them to full-text screening. These articles mainly came from the Zoological Records database (93\% of them). This may explain why our review contained few articles from the Zoological Records database (21 out of 119 articles). Nevertheless, most of the articles for which we did not find the full text were also from the Zoological Records database (169 out of the 209 articles with missing full text), suggesting that many of these excluded references could not have been included in the review in the end. We however made the list of these excluded articles easily accessible for possible further use (Additional file 11). We also recommend that managers of publication databases pay attention to this issue of lacking abstract because it can interfere with the proper conduct of systematic reviews as searching for full-text and full-text screening are time-consuming steps. On the other hand, although it is a good practice, we were unable to test the consistency of screeners' decisions at full-text screening due to logistical constraints and time limitation. Clarifications and adjustments between screeners were made before the full-text assessment, and a retrospective check of a random sample of $10 \%$ of the articles rejected was done. This check revealed that it seems unlikely that some articles were missed because of inconsistency between screeners' decisions. In addition, as we included only articles with full-text written in English or French, included studies are biased towards English and French speaking countries. The list of articles rejected because of language is nevertheless easily accessible for possible further use in Additional file 5. Last, our searches for grey literature were not consistent between the beginning of the review (call for grey literature) and the update for literature (searches on specialist websites and on BASE and CORE search engines). The call for literature made at the beginning of the review was very time-consuming for few included studies, which is why we changed search strategy to update the grey literature.

Second, there is a set of limitations linked to the experimental design of the studies included in our review. We found few studies based on long term (more than 2 years) data and the conclusions of the individual studies may thus be influenced by inter-annual variations in 
population sizes. The seasonal dimension was also not always taken into account in protocols, while several studies showed statistically significant variations in their results depending on the season considered [57, 79, 112, 116]. The choice of the measurement period may be of particular importance for the specific question Q1 on the influence of management practices on biodiversity in verges. For instance, in a study assessing bird response to planting fleshly-fruited woody species on highway verges, measurements were performed only 1 year after the intervention, and no effect of the intervention on bird communities was found [118]. In this case, the lack of effects may be due to a delayed response of bird communities to the intervention, which was not considered in the protocol of the study. In addition, only two studies included in this review incorporate data collection before the intervention. This can be problematic to evaluate the influence of management practices, because it is not possible to distinguish real management effect from pre-existing differences between control and managed sites. Last, it should be noted that different proxies for abundance were measured in the studies (density, occurrence, activity, ...) and all were considered equivalent in our analysis. This means that an individual making temporary use of a verge for foraging (e.g. a bat) has the same amount of meaning as an individual living in a verge (e.g. a small mammal).

Third, the conclusions about waterways may be limited due to the difficulty in assessing waterway navigability. Indeed, the navigability of waterways is rarely reported by the authors and there is no international database that refers to this information. Thus, we included articles with Strahler [26] stream order above three, canals and rivers (Table 5) because these criteria sound relevant to restrict included articles to major waterways, implicitly assuming that they might be navigable. We are aware that this solution is not perfect: some articles about navigable waterways could have been rejected and others about non-navigable waterways could have been included.

Fourth, we limited our narrative syntheses to the comparisons that were statistically tested. This resulted in the exclusion of 37 studies, often because they compared more than two categories and did not provide results for each comparison (e.g. Kruskal-Wallis test or ANOVA without post hoc tests). From these 37 excluded studies, six however provided appropriate data that were included in the meta-analyses.

Fifth, for five of our six specific questions the small number of studies as well as their lack of variable homogeneity limited our ability to implement meta-analyses (e.g. eight different types of interventions for the specific question Q1 that gathered 21 studies). These studies were included in the review in narrative syntheses, but narrative syntheses cannot provide reliable information on the magnitude of the response (effect size) and on whether this magnitude is consistent across studies [167]. Also, the meta-analyses assessing the effect of exposition to railways and powerlines on vertebrate abundance were performed on a low number of cases $(\mathrm{N}=11$ and $\mathrm{N}=8$ for powerlines and railways, respectively). The power of the tests was consequently relatively low, which may explain why statistically significant effects were not identified. In addition, the meta-analyses performed on abundance included effect sizes computed with both individual species abundance (species-level abundance) and abundance of a group of species (community-level abundance). This means that we gave a similar weight in the analysis to the change in abundance of a species, which could be a sensitive one because single-species studies often focus on species of conservation concern, and to the change in the mean abundance of a group of species, where common, less sensitive species, could mask the results for sensitive ones. We performed separate additional analyses for abundance at community level and at species level (Additional file 12) that did not challenge our conclusions but sample size was too limited to be able to really assess whether this bias effectively occurred.

Sixth, the small number of studies retrieved for the specific questions related to management practices may arise from the design of our search strings. Indeed, the search strings we used did not include specific terms related to management practices such as "road lighting" or "mowing" (Table 2) and we may thus have missed potential studies having keywords related to management practices and to LTIs without any keywords related to outcomes or verges.

Finally, we noticed an exponential increase in literature about our primary review question (Fig. 3). It should be noted that the content of Web Of Science also increased exponentially over time. This implies that this systematic review on vertebrates should be regularly updated. Indeed, our review was highly skewed towards roads, birds and small mammals, and our conclusions about other LTIs and biological groups should be confirmed by the inclusion of future studies. As the review focused on vertebrates, we would like to emphasize that this review only represents a partial estimate of the potential of LTI verges as habitat and/or corridor for biodiversity (but see Villemey et al. [22] for insects).

\section{Review conclusions}

\section{Implications for policy/management}

We found that the potential of linear transportation infrastructure (LTI) verges to constitute a habitat for vertebrate species varies according to the infrastructure and the biological group considered. Relatively natural 
waterways could be positive for the abundance of birds and bats, highways can benefit to small mammals but seem detrimental to passerine birds, and road verges can constitute a corridor for some specific mammal species. Our results should be taken with caution by LTI managers, as our review did not assess the negative impacts that the infrastructure itself could have on the species living in verges. Indeed, living in verges could be at risk for species, and verges could be attractive in some aspects but ultimately become unsuitable for survival or reproduction (i.e. become ecological trap). For instance, we found that Tasmanian devils in Australia were more likely to move along roads than to move along other linear features [119], seemingly because as scavenger specialists they find carcasses of animals killed by vehicles. But they are themselves at high risk of mortality due to collision with vehicles, causing a decrease in population size [168].

In addition, the presence of a species on verges should be contextualised at a larger scale as it may disturb species population dynamics. For instance, we found that roads lit with white lamps support greater bat activity than roads lit with orange lamps or unlit roads, but this should not be considered positive for the species. Even if at first sight some bat species may benefit from road lighting to forage insects attracted by light, populationlevel analyses at the landscape-scale actually revealed a negative effect of lighting, probably because lighting does not only influence foraging behaviour but also reproduction and commuting behaviours [169]. Interactions between species should also be considered because the presence of a species on verges may have indirect negative effect on other species. For instance, the probability of a predator being killed on the road may be related to the presence of its prey on road verges [133, 170]. We thus recommend LTI managers willing to manage verges to enhance the presence of particular species to gather information on their risk of mortality due to the LTI, their interaction with other species, and their overall population status in the area, that is, to adopt ecosystem and landscape levels approaches.

Finally, the appropriate management of verges for compensating the loss of biodiversity generated by LTIs is a challenge, as the response may depend on the management practice, species characteristics, the type of habitat in the verges and landscape context, with interactions between all these factors. However, this challenge must be met as verges have a strong potential to contribute substantially to biodiversity loss mitigation in a context of constant loss of natural habitats.

\section{Implications for research}

Given that LTI managers have most interest in knowing the potential of linear transportation infrastructure (LTI) verges as a corridor for biodiversity and that we found few studies addressing this question we advise to dedicate resources on the monitoring of vertebrate movements on verges. The systematic review on insect biodiversity on LTI verges led to the same recommendation, with even fewer studies on the role of corridor of verges for insects (2 studies) than for vertebrates (7). Also, for vertebrates, when they exist the studies rarely directly measured movements on verges. We thus encourage more research on this topic and the development of protocols that enable direct measure of the longitudinal movements on verges, and that use appropriate comparators. Assessing the role of LTI verges as corridors for biodiversity has also implications for research on invasive species.

Moreover, as the appropriate management of verges for biodiversity loss mitigation is a challenge, the effect of management practices on the role of habitat of verges should deserve further investigation. A coordination of research efforts on one specific management practice (e.g. vegetation management) as well as common protocols should be implemented to be able to perform quantitative syntheses (meta-analyses) and test the effect of this management practice.

We also advocate the integration of the seasonal dimension in protocols aiming at assessing biodiversity in verges. We indeed found several studies showing statistically significant variations in their results depending on the season considered (e.g. [57, 79, 112, 116]). Sampling may focus on a key season (e.g. breeding season) or may be replicated for all seasons.

More generally, we encourage protocols with true replication and systematic or random sampling (only $39 \%$ of the studies included in this review had these two characteristics) so that the results could be generalized. We also encourage protocols with several measures in time so that long-term effects could be assessed. A recent simulation study also demonstrated that designs other than Before-After-Control-Impact may lead to biased estimates of the true effect of an environmental impact, even when the sample size is large [171]. This kind of protocols requires consequent funding investment and is relatively difficult to implement in ecology but leads to robust results able to inform decisions-makers. We also encourage the formalisation of protocols leading to robust results into guidelines and standards to inform both researchers and funders.

Finally, to further facilitate the use of data in metaanalyses we advocate a common effort of the research community to indicate in the tables and figures of published or unpublished papers the number of observations along with the name of the computed statistics (e.g. mean) and of the measure of variance (e.g. standard deviation). More generally, we recommend authors, editors, 
and peer-reviewers to pay more attention on the description of the methods because articles often fail to provide sufficient methodological details to allow precise understanding, which limits their inclusion in evidence synthesis [172].

\section{Supplementary information}

Supplementary information accompanies this paper at https://doi. org/10.1186/s13750-020-00196-7.

Additional file 1. ROSES systematic review checklist. ROSES form for systematic review reports version 1.0.

Additional file 2. Test list. List of the 102 articles used to assess the comprehensiveness of the search with information on the method to get them and on their indexation in publication databases and retrieval with the search string

Additional file 3. Search strings used for search engines. Search strings that were used for search engines Google Scholar, BASE, and CORE.

Additional file 4. Searches for literature summary. Summary of all the searches for literature with dates of search and number of articles found.

Additional file 5. Articles excluded at full-text. List of articles excluded at full-text screening with their reasons for exclusion.

Additional file 6. Study validity assessment. List of the studies included in the review with study validity assessment. For the studies excluded from further synthesis after critical appraisal, a short explanation of the reason for exclusion is provided.

Additional file 7. Systematic review table. List of the studies included in the review with coded metadata.

Additional file 8. Narrative tables. Tables summarizing key results of the studies included in the narrative syntheses.

Additional file 9. Meta-analyses database. Table of all the variables extracted for computing effect sizes and analysing reasons for heterogeneity in effect sizes, and value and variance of the effect sizes computed.

Additional file 10. Publication bias analysis. Analysis of publication bias with funnel plot, Egger's regression test and cumulative meta-analysis.

Additional file 11. List of excluded without-abstract articles. List of the articles that were excluded because they did not have an abstract.

Additional file 12. Separated meta-analyses on abundance measured at community and species levels. Summary flow chart and results of the meta-analyses performed separately on abundance measured at community level and at species level.

\section{Acknowledgements}

We thank the CILB, the French Ministry of Ecology and the ADEME for funding this research project, the ITTECOP research incentive program for their logistical help and the FRB for their methodological support on systematic reviews and the organization of the steering committee meetings. We thank Barbara Livoreil (FRB) who contributed to the validation of the control list, the database selection, the search terms brainstorming and the external experts' workshop for critical appraisal; Patrick Pacevicius (Cerema) for his contribution to the screening of articles from the call to subject experts and Google Scholar; Véronique Roy (MNHN), Nadine Deniaud (MNHN), and Emmanuel Jaslier (Université Paris 6 Pierre et Marie Curie) for their contribution to the selection of databases and search equation and support for the bibliographical stage of the study; and Magali Chao $(\mathrm{MNHN})$ for her help in searching the full text of articles. We also thank Leyli Borner and Isabelle Le Viol (MNHN), Jean Carsignol (Cerema), Violette Le Féon (Institut national de la recherche agronomique), Jean-François Godeau (Life Elia project), Sébastien Filoche (Conservatoire botanique national du Bassin parisien) and Damien Picard (Université d'Angers) for their contribution to the critical appraisal workshop. Finally, we thank the "Union internationale pour la conservation de la nature France", the French Ministry of Ecology, the "Fédération des Parcs Naturels
Régionaux", the "Centre d'études et d'expertise sur les risques, l'environnement, la mobilité et l'aménagement", the "Société Anonyme Française d'Étude de Gestion et d'Entreprises", the "Société d'études techniques et économiques", the members of the ITTECOP program, the CILB and all the contacted experts for sending us scientific and grey literature and giving us contact of other experts. We also thank three anonymous reviewers for their helpful comments on the manuscript.

\section{Other projects}

After responding to a call for tender, the MNHN was selected to conduct another project than the present one, named Trans-fer, which was also funded by Réseau Ferré de France (a member of the CILB) and the French Ministry of Ecology. Trans-fer ended before the beginning of the present project and the two projects were steered independently.

\section{Authors' contributions}

$\mathrm{RS}, \mathrm{YB}, \mathrm{AC}$ and JT conceived the review question. AJ, MV, RS and SV undertook the experts request for scientific key articles. AJ, RS, MV, AC, YB and SV validated the control list. MV, FFDL, AJ, RS, AC, YB and SV contributed to the database selection. AJ, MV, RS, AC, YB, SV and FFDL contributed to the search terms brainstorming. AJ, MV, RS and FFDL undertook the search comprehensiveness assessment and equation selection. RS and AJ organized the external experts' workshop for critical appraisal and AC, YB, SV attended it. Literature stemming from Web of Science and Zoological Records was screened on title, summary and full text by AJ, RS, MV, SV, AC, YB, EG, ELM, VR, AV, YR, and DYO. Literature stemming from the call to subject experts was screened on title by MV and on full-text by EG, ELM and VR. Literature stemming from Google Scholar was screened on title and full-text by EG, ELM, VR, RS, and DYO. Critical appraisal was made by VA, MH, and DYO, and extraction of the results in narrative tables was made by DYO and reviewed by AC. DYO performed data extraction for the meta-analyses. DYO and IW implemented the meta-analyses and $\mathrm{HJ}$ offered his support on specific questions. DYO, RS, and YR designed the analyses. RS was the scientific coordinator of the project. FFDL offered support for the bibliographical stage of the study. All authors joined regular steering committee meetings. DYO and AV wrote the manuscript. All authors read and approved the final manuscript.

\section{Funding}

The project was supported by the CILB, the French Ministry of Ecology, the ADEME and the FRB. It was funded by the CILB, which is composed of the following LTI managing organizations: Réseau Ferré de France (now named SNCF Réseau), Voies Navigables de France, Réseau de Transport d'Électricité, GRT Gaz, Transport et Infrastructures Gaz France and Électricité Réseau Distribution France (now ENEDIS), EIFFAGE and Association des Sociétés Françaises d'Autoroutes. Besides, the French Ministry of Ecology and the ADEME supported the project through the ITTECOP program which constituted a framework for the project, offered a partial financial contribution to the project and provided funding through the Cerema participation. Finally, the FRB gave the research team methodological support for the realization of the systematic review.

\section{Availability of data and materials}

All data generated or analysed during this study are included in this published article and its additional files.

\section{Ethics approval and consent to participate}

Not applicable.

\section{Consent for publication}

Not applicable.

\section{Competing interests}

The authors declare that they have no competing interests.

\section{Author details}

1 UMS PatriNat, OFB - MNHN - CNRS, 75005 Paris, France. ${ }^{2}$ INRAE PACA, centre Aix-en-Provence, Aix-en-Provence, France. ${ }^{3}$ Centre d'Ecologie et de Sciences de la COnservation (CESCO), Muséum National d'Histoire Naturelle - Centre National de la Recherche Scientifique - Sorbonne Université, 75005 Paris, France. ${ }^{4}$ CEFE, CNRS - EPHE - IRD - Univ Montpellier - Univ Paul Valéry Montpellier 3, Montpellier, France. ${ }^{5}$ Centre d'études et d'Expertises sur les 
Risques, I'Environnement, la Mobilité et l'Aménagement (Cerema) Sud-Ouest, Saint-Médard-en-Jalles, France. ${ }^{6}$ SPE, INRAE, 78026 Versailles, France. ${ }^{7}$ Univ. Bordeaux, 33405 Talence, France. ${ }^{8}$ BIOGECO, INRAE - University of Bordeaux, 33610 Cestas, France.

Received: 28 November 2019 Accepted: 25 May 2020 Published online: 05 June 2020

\section{References}

1. Butchart SHM, Walpole M, Collen B, van Strien A, Scharlemann JPW, Almond REA, et al. Global biodiversity: indicators of recent declines. Science. 2010;328:1164-8.

2. Maxwell SL, Fuller RA, Brooks TM, Watson JEM. Biodiversity: the ravages of guns, nets and bulldozers. Nat News. 2016;536:143.

3. Bekker $\mathrm{H}$, luell B. Habitat fragmentation due to infrastructure. Making Connections. 2003;15.

4. Biasotto LD, Kindel A. Power lines and impacts on biodiversity: a systematic review. Environ Impact Assess Rev. 2018;71:110-9.

5. Dorsey B, Olsson M, Rew LJ. Ecological effects of railways on wildlife. In: Van Der Ree R, Smith DJ, Grilo C, editors. Handbook of road ecology. Hoboken: Wiley; 2015. p. 219-27. https://doi.org/10.1002/9781118568 170.ch26.

6. Jaeger JAG, Soukup T, Schwick C, Madriñan LF, Kienast F. Lanscape fragmentation in Europe. European landscape dynamics corine land cover data. Jan Feranec, Tomas Soukup, Gerard Hazeu, and Gabriel Jaffrain. Boca Raton: CRC Press; 2016.

7. Richardson ML, Wilson BA, Aiuto DA, Crosby JE, Alonso A, Dallmeier F, et al. A review of the impact of pipelines and power lines on biodiversity and strategies for mitigation. Biodivers Conserv. 2017:26:1801-15.

8. Trombulak SC, Frissell CA. Review of ecological effects of roads on terrestrial and aquatic communities. Conserv Biol. 2000;14:18-30.

9. Krauss J, Bommarco R, Guardiola M, Heikkinen RK, Helm A, Kuussaari $M$, et al. Habitat fragmentation causes immediate and time-delayed biodiversity loss at different trophic levels. Ecol Lett. 2010;13:597-605.

10. Benitez-Lopez A, Alkemade R, Verweij PA. The impacts of roads and other infrastructure on mammal and bird populations: a meta-analysis. Biol Conserv. 2010;143:1307-16.

11. Zachos FE, Althoff C, Steynitz YV, Eckert I, Hartl GB. Genetic analysis of an isolated red deer (Cervus elaphus) population showing signs of inbreeding depression. Eur J Wildl Res. 2007:53:61-7.

12. Seiler A. Effects of infrastructure on nature. In: Trocmé M, Cahill S, De Vries JG, Farrall H, Folkeson L, Fry G, Hicks C, Peymen J, editors. COST action 341-habitat fragmentation due to transportation infrastructure: the European review. Luxembourg: Office for Official Publications of the European Communities; 2002. p. 31-50.

13. Bolger DT, Scott TA, Rotenberry JT. Use of corridor-like landscape structures by bird and small mammal species. Biol Conserv. 2001;102:213-24

14. Forman RTT, Alexander LE. Roads and their major ecological effects. Annu Rev Ecol Syst. 1998;29:207-31.

15. Le Viol I, Julliard R, Kerbiriou C, de Redon L, Carnino N, Machon N, et al. Plant and spider communities benefit differently from the presence of planted hedgerows in highway verges. Biol Conserv. 2008;141:1581-90.

16. Bennett AF. Linkages in the landscape: the role of corridors and connectivity in wildlife conservation. Gland: IUCN; 2003.

17. Gilbert-Norton L, Wilson R, Stevens JR, Beard KH. A meta-analytic review of corridor effectiveness. Conserv Biol. 2010;24:660-8.

18. Heller NE, Zavaleta ES. Biodiversity management in the face of climate change: a review of 22 years of recommendations. Biol Conserv. 2009; 142:14-32.

19. Fahrig $L$, Rytwinski T. Effects of roads on animal abundance: an empirical review and synthesis. Ecol Soc. 2009;14. https://ir.library.carleton.ca/ pub/4484. Accessed 14 Oct 2019.

20. Pullin AS, Stewart GB. Guidelines for systematic review in conservation and environmental management. Conserv Biol. 2006;20:1647-56.

21. Jeusset A, Vargac M, Bertheau Y, Coulon A, Deniaud N, Flamerie De Lachapelle F, et al. Can linear transportation infrastructure verges constitute a habitat and/or a corridor for biodiversity in temperate landscapes? A systematic review protocol. Environ Evid. 2016;5:5.
22. Villemey A, Jeusset A, Vargac M, Bertheau Y, Coulon A, Touroult J, et al. Can linear transportation infrastructure verges constitute a habitat and/ or a corridor for insects in temperate landscapes? A systematic review. Environ Evid. 2018;7:5.

23. Phillips BB, Gaston KJ, Bullock JM, Osborne JL. Road verges support pollinators in agricultural landscapes, but are diminished by heavy traffic and summer cutting. J Appl Ecol. 2019;56:2316-27.

24. Collaboration for Environmental Evidence. Guidelines and standards for evidence synthesis in environmental management. Version 5.0. In: Pullin AS, Frampton GK, Livoreil B, Petrokofsky G, editors. http://www.envir onmentalevidence.org/information-for-authors. Accessed 12 Nov 2019.

25. Haddaway N, Macura B, Whaley P, Pullin A. ROSES flow diagram for systematic reviews. Version 1.0. 2018. https://figshare.com/articles/ ROSES_Flow_Diagram_Version_1_0/5897389.

26. Strahler AN. Quantitative analysis of watershed geomorphology. Trans Am Geophys Union. 1957;38:913-20.

27. Lajeunesse MJ. Recovering missing or partial data from studies: a survey of conversions and imputations for meta-analysis. In: Koricheva J, Gurevitch J, Mengersen K, editors. Handbook of meta-analysis in ecology and evolution. Princeton: Princeton University Press; 2013. p. 195-206.

28. Higgins JPT, White IR, Anzures-Cabrera J. Meta-analysis of skewed data: combining results reported on log-transformed or raw scales. Stat Med. 2008;27:6072-92.

29. Adams LW, Geis AD. Effects of roads on small mammals. J Appl Ecol. 1983;20:403-15.

30. Ben-Ami D, Ramp D. Impact of roadside habitat on swamp wallaby movement and fitness. Wildl Res. 2013;40:512-22.

31. Berthinussen A, Altringham J. The effect of a major road on bat activity and diversity. J Appl Ecol. 2012;49:82-9.

32. Bissonette JA, Rosa SA. Road zone effects in small-mammal communities. Ecol Soc. 2009;14:27.

33. Da Silva VP, Deffaci AC, Hartmann MT, Hartmann PA. Birds around the road: effects of a road on a savannah bird community in southern Brazil. Ornitol Neotrop. 2017;28:119-28.

34. Goosem M, Marsh H. Fragmentation of a small-mammal community by a powerline corridor through tropical rainforest. Wildl Res. 1997;24:613-29.

35. Maltchik L, Peixoto CD, Stenert C, Moreira LFB, Mochado IF. Dynamics of the terrestrial amphibian assemblage in a flooded riparian forest fragment in a neotropical region in the south of Brazil. Braz J Biol. 2008;68:763-9.

36. Meunier FD, Verheyden C, Jouventin P. Use of roadsides by diurnal raptors in agricultural landscapes. Biol Conserv. 2000;92:291-8.

37. Petit LJ, Petit DR. Factors governing habitat selection by prothonotary warblers: field tests of the Fretwell-Lucas models. Ecol Monogr. 1996;66:367-87.

38. Takatsuki S. A case study on the effects of a transmission-line corridor on Sika deer habitat use at the foothills of Mt Goyo, northern Honshu, Japan. Ecol Res. 1992;7:141-6.

39. Hedges LV, Olkin I. Statistical methods for meta-analysis. Orlando: Academic Press; 1985.

40. Hedges LV. Distribution theory for glass's estimator of effect size and related estimators. J Educ Stat. 1981;6:107-28.

41. Hamman EA, Pappalardo P, Bence JR, Peacor SD, Osenberg CW. Bias in meta-analyses using Hedges' d. Ecosphere. 2018;9:e02419.

42. Hedges LV. Estimation of effect size from a series of independent experiments. Psychol Bull. 1982;92:490-9.

43. Rosenberg MS. The file-drawer problem revisited: a general weighted method for calculating fail-safe numbers in meta-analysis. Evolution. 2005:59:464-8

44. Nakagawa S, Santos ESA. Methodological issues and advances in biological meta-analysis. Evol Ecol. 2012;26:1253-74.

45. Egger M, Davey Smith G, Schneider M, Minder C. Bias in meta-analysis detected by a simple, graphical test. BMJ. 1997;315:629-34.

46. R Core Team. R: a language and environment for statistical computing. Vienna: R Foundation for Statistical Computing; 2019. https://www.Rproject.org/.

47. Viechtbauer W. Conducting meta-analyses in R with the metafor package. J Stat Softw. 2010;36:1-48.

48. Rich AC, Dobkin DS, Niles LJ. Defining forest fragmentation by corridor width: the influence of narrow forest-dividing corridors 
on forest-nesting birds in southern New Jersey. Conserv Biol. 1994;8:1109-21.

49. Webb WC, Marzluff JM, Hepinstall-Cymerman J. Linking resource use with demography in a synanthropic population of common ravens. Biol Conserv. 2011;144:2264-73.

50. Weiermans J, van Aarde RJ. Roads as ecological edges for rehabilitating coastal dune assemblages in northern KwaZulu-Natal, South Africa. Restor Ecol. 2003;11:43-9.

51. Ascensao F, Clevenger AP, Grilo C, Filipe J, Santos-Reis M. Highway verges as habitat providers for small mammals in agrosilvopastoral environments. Biodivers Conserv. 2012;21:3681-97.

52. Rotholz E, Mandelik Y. Roadside habitats: effects on diversity and composition of plant, arthropod, and small mammal communities. Biodivers Conserv. 2013;22:1017-31.

53. Stewart RA, ClarkTJ, Shelton J, Stringfellow M, Scott C, White SA, et al. Urban grasslands support threatened water voles. J Urban Ecol. 2017;3. https://academic.oup.com/jue/article/3/1/jux007/4097929. Accessed 13 Sept 2018

54. Pescador M, Peris S. Influence of roads on bird nest predation: an experimental study in the Iberian Peninsula. Landsc Urban Plan. 2007:82:66-71.

55. Delgado JD, Arevalo JR, Fernandez-Palacios JM. Road and topography effects on invasion: edge effects in rat foraging patterns in two oceanic island forests (Tenerife, Canary Islands). Ecography. 2001;24:539-46.

56. Jumeau J, Boucharel P, Handrich Y, Burel F. Road-related landscape elements as a habitat: a main asset for small mammals in an intensive farming landscape. Basic Appl Ecol. 2017;25:15-27.

57. Meunier FD, Corbin J, Verheyden C, Jouventin P. Effects of landscape type and extensive management on use of motorway roadsides by small mammals. Can J Zool. 1999;77:108-17.

58. Santos SM, Mathias MdL, Mira A, Simoes MP. Vegetation structure and composition of road verge and meadow sites colonized by cabrera vole (Microtus Cabrerae Thomas). Pol J Ecol. 2007;55:481-93.

59. Bechet A, Isenmann P, Gaudin R. Nest predation, temporal and spatial breeding strategy in the Woodchat Shrike Lanius senator in Mediterranean France. Acta Oecol. 1998;19:81-7.

60. Delgado JD, Arevalo JR, Maria Fernandez-Palacios J. Bird communities in two oceanic island forests fragmented by roads on Tenerife, Canary Islands. Ostrich. 2008;79:219-26.

61. Geerts S, Pauw A. Easy technique for assessing pollination rates in the genus Erica reveals road impact on bird pollination in the Cape fynbos, South Africa. Austral Ecol. 2011:36:656-62.

62. Holbrook JD, Vierling KT, Vierling LA, Hudak AT, Adam P. Occupancy of red-naped sapsuckers in a coniferous forest: using LiDAR to understand effects of vegetation structure and disturbance. Ecol Evol. 2015:5:5383-93.

63. Holm TE, Laursen K. Car traffic along hedgerows affects breeding success of Great Tits Parus major. Bird Study. 2011;58:512-5.

64. Meunier FD, Verheyden C, Jouventin P. Bird communities of highway verges: influence of adjacent habitat and roadside management. Acta Oecol. 1999;20:1-13.

65. Weakland CA, Wood PB. Cerulean Warbler (Dendroica cerulea) microhabitat and landscape-level habitat characteristics in southern west Virginia. Auk. 2005;122:497-508.

66. Yamac E, Kirazli C. Road Effect on the breeding success and nest characteristics of the Eurasian Magpie (Pica pica). Ekoloji. 2012;21:1-10.

67. Hindmarch S, Elliott JE, McCann S, Levesque P. Habitat use by barn owls across a rural to urban gradient and an assessment of stressors including, habitat loss, rodenticide exposure and road mortality. Landsc Urban Plan. 2017:164:132-43.

68. Scarpignato AL, George TL. Space use by common ravens in marbled murrelet nesting habitat in Northern California. J Field Ornithol. 2013;84:147-59.

69. Myczko L, Sparks TH, Skorka P, Rosin ZM, Kwiecinski Z, Gorecki MT, et al. Effects of local roads and car traffic on the occurrence pattern and foraging behaviour of bats. Transp Res Part D Transp Environ. 2017:56:222-8.

70. Mallard F. Chapitre IV. Etudes de sensibilité de quatre indicateurs biotiques potentiels des habitats forestiers. Développement d'une méthode d'évaluation quantitative des effets des projets d'infrastructures de transport terrestre sur les milieux naturels. 2014.
71. Claireau F. Chapitre 2. Effets des autoroutes sur l'activité chiroptérologique et la génétique des populations (Article $n^{\circ} 3$ ). Evaluation des impacts de la fragmentation autoroutière sur les chauves-souris à différentes échelles spatio-temporelles. 2018. http://www.theses.fr/s1491 11. Accessed 6 Déc 2018.

72. Hein CD, Castleberry SB, Miller KV. Site-occupancy of bats in relation to forested corridors. For Ecol Manage. 2009;257:1200-7.

73. O'Donnell CFJ, Christie JE. Habitat use and nocturnal activity of lesser short-tailed bats (Mystacina tuberculata) in comparison with long-tailed bats (Chalinolobus tuberculatus) in temperate rainforest. N Z J Zool. 2006;33:113-24.

74. Waters D, Jones G, Furlong M. Foraging ecology of Leisler's bat (Nyctalus leisleri) at two sites in southern Britain. J Zool. 1999;249:173-80.

75. Owen SF, Berl JL, Edwards JW, Ford WM, Wood PB. Raccoon Spatial Requirements and Multi-Scale Habitat Selection within an Intensively Managed Central Appalachian Forest. Am Midl Nat. 2015;174:87-95.

76. Niu HY, Xing JJ, Zhang HM, Wang D, Wang XR. Roads limit of seed dispersal and seedling recruitment of Quercus chenii in an urban hillside forest. Urban For Urban Green. 2018;30:307-14.

77. Akmali V, Sharifi M, Farassat H. Habitat selection by the common pipistrelle, Pipistrellus pipistrellus s. I. (Chiroptera: Vespertilionidae), in the Dinevar region of western Iran. Zool Middle East. 2004;33:43-50.

78. Anderson J, Law B, Tidemann C. Stream use by the large-footed myotis Myotis macropus in relation to environmental variables in northern New South Wales. Aust Mammal. 2006:28:15-26.

79. Barros MAS, Pessoa DMA, Rui AM. Habitat use and seasonal activity of insectivorous bats (Mammalia: Chiroptera) in the grasslands of southern Brazil. Zoologia. 2014;31:153-61.

80. Clear S. Factors affecting the distribution of Pipistrellus pipistrellus and Pipistrellus pygmaeus in the Lothians region, Scotland. BaTML Publications. 2005;2:23-33.

81. Ellis AM, Patton LL, Castleberry SB. Bat activity in upland and riparian habitats in the Georgia piedmont. In: Proceedings of the annual conference SEAFWA. vol. 56. 2002. p. 210-8.

82. Lloyd A, Law B, Goldingay R. Bat activity on riparian zones and upper slopes in Australian timber production forests and the effectiveness of riparian buffers. Biol Conserv. 2006;129:207-20.

83. Rachwald A, Bradford T, Borowski Z, Racey PA. Habitat preferences of soprano pipistrelle Pipistrellus pygmaeus (Leach, 1825) and common pipistrelle Pipistrellus pipistrellus (Schreber, 1774) in two different woodlands in North East Scotland. Zool Stud. 2016;55.

84. Law BS, Chidel M, Tap P. Bat activity in ephemeral stream-beds in the Pilliga forests: clarifying the importance of flyways and buffer widths in open forest and woodland. The biology and conservation of Australasian bats. R Zool Soc N S W. 2011. https://doi.org/10.7882/FS.2011.031.

85. Gatesire T, Nsabimana D, Nyiramana A, Seburanga JL, Mirville MO. Bird diversity and distribution in relation to urban landscape types in Northern Rwanda. Sci World J. 2014;157824:1-12.

86. Lock PA, Naiman RJ. Effects of stream size on bird community structure in coastal temperate forests of the Pacific Northwest, USA. J Biogeogr. 1998;25:773-82.

87. Rabearivony J, Brady LD, Jenkins RK, Griffiths RA, Raselimanana AP, Bisoa $M$, et al. Influence of riparian habitats on the distribution of rainforest chameleons in Parc National de Ranomafana, Madagascar. Afr J Herpetol. 2015;64:148-59.

88. Evans DR, Gates JE. Cowbird selection of breeding areas: the role of habitat and bird species abundance. Wilson Bull. 1997;109:470-80.

89. Tryjanowski P, Sparks TH, Jerzak L, Rosin ZM, Skórka P. A paradox for conservation: electricity pylons may benefit avian diversity in intensive farmland. Conserv Lett. 2014;7:34-40.

90. Clarke DJ, Pearce KA, White JG. Powerline corridors: degraded ecosystems or wildlife havens? Wildl Res. 2006;33:615-26.

91. Doherty PF, Grubb TC. Reproductive success of cavity-nesting birds breeding under high-voltage powerlines. Am Midl Nat. 1998;140:122-8.

92. Castillo E, Priotto J, Ambrosio AM, Provensal MC, Pini N, Morales MA, et al. Commensal and wild rodents in an urban area of Argentina. Int Biodeterior Biodegrad. 2003;52:135-41.

93. Cerboncini RAS, Roper JJ, Passos FC. Edge effects without habitat fragmentation? Small mammals and a railway in the Atlantic Forest of southern Brazil. Oryx. 2016;50:460-7. 
94. León VA, Fraschina J, Guidobono JS, Busch M. Habitat use and demography of Mus musculus in a rural landscape of Argentina. Integr Zool. 2013;8(Suppl 1):18-29.

95. Vandevelde J-C, Bouhours A, Julien J-F, Couvet D, Kerbiriou C. Activity of European common bats along railway verges. Ecol Eng. 2014;64:49-56.

96. Janiszewski T, Minias P, Wojciechowski Z. Selective forces responsible for transition to nesting on electricity poles in the White Stork Ciconia ciconia. Ardea. 2015;103:39-50.

97. Todd VLG, Waters DA. Small scale habitat preferences of Myotis daubentonii, Pipistrellus pipistrellus, and potential aerial prey in an upland river valley. Acta Chiropterol. 2017;19:255-72.

98. Lintott PR, Bunnefeld N, Park KJ. Opportunities for improving the foraging potential of urban waterways for bats. Biol Conserv. 2015;191:224-33.

99. Lopez-Baucells A, Casanova L, Puig-Montserrat X, Espinal A, Paramo F, Flaquer C. Evaluating the use of Myotis daubentonii as an ecological indicator in Mediterranean riparian habitats. Ecol Ind. 2017;74:19-27.

100. Langton SD, Briggs PA, Haysom KA. Daubenton's bat distribution along rivers - developing and testing a predictive model. Aquat Conserv Marine Freshw Ecosyst. 2010;20:545-54.

101. Scher O, Thièry A. Odonata, amphibia and environmental characteristics in motorway stormwater retention ponds (Southern France). Hydrobiologia. 2005;551:237-51.

102. Askins RA, Folsom-O'Keefe CM, Hardy MC. Effects of vegetation, corridor width and regional land use on early successional birds on powerline corridors. PLOS ONE. 2012;7:e31520.

103. Adams LW. Small mammal use of an interstate highway median strip. J Appl Ecol. 1984;21:175-8.

104. Bellamy PE, Shore RF, Ardeshir D, Treweek JR, Sparks TH. Road verges as habitat for small mammals in Britain. Mammal Rev. 2000;30:131-9.

105. Clarke DJ, White JG. Recolonisation of powerline corridor vegetation by small mammals: timing and the influence of vegetation management. Landsc Urban Plan. 2008;87:108-16.

106. Vaughan IP, Noble DG, Ormerod SJ. Combining surveys of river habitats and river birds to appraise riverine hydromorphology. Freshw Biol. 2007:52:2270-84.

107. Vilches A, Miranda R, Arizaga J, Galicia D. Habitat selection by breeding common kingfishers (Alcedo atthis L.) in rivers from Northern Iberia. Ann Limnol Int J Limnol. 2012;48:289-94.

108. Zabala J, Zuberogoitia I, Martinez-Climent JA. Factors affecting occupancy by the European mink in south-western Europe. Mammalia. 2006;70:193-201.

109. Chen X. Distribution patterns of invasive alien species in Alabama, USA. Manage Biol Invasions. 2012;3:25-36.

110. Moffatt KC, Crone EE, Holl KD, Schlorff RW, Garrison BA. Importance of hydrologic and landscape heterogeneity for restoring bank swallow (Riparia riparia) colonies along the Sacramento River, California. Restor Ecol. 2005;13:391-402.

111. Royan A, Hannah DM, Reynolds SJ, Noble DG, Sadler JP. Avian community responses to variability in river hydrology. PLOS ONE. 2013;8:e83221.

112. Farago S, Hangya K. Effects of water level on waterbird abundance and diversity along the middle section of the Danube River. Hydrobiologia. 2012;697:15-21.

113. Day J, Baker J, Schofield H, Mathews F, Gaston KJ. Part-night lighting: implications for bat conservation. Anim Conserv. 2015;18:512-6.

114. Roche N, Langton S, Aughney T. Car-based bat monitoring in Ireland 2003-2011. Irish Wildl Man. 2012.

115. Blake D, Hutson AM, Racey PA, Rydell J, Speakman JR. Use of lamplit roads by foraging bats in southern England. J Zool. 1994;234:453-62.

116. Campbell MO. The impact of vegetation, river, and urban features on waterbird ecology in Glasgow, Scotland. J Coast Res. 2008:24:239-45.

117. Fox A. The role of habitat enhancement in the return of the European otter (Lutra lutra) to Northumberland. J Chart Inst Water Environ Manage. 1999;13:79-83.

118. de Torre R, Jimenez MD, Ramirez A, Mola I, Casado MA, Balaquer L. Use of restoration plantings to enhance bird seed dispersal at the roadside: failures and prospects. J Environ Eng Landsc Manage. 2015;23:302-11.

119. Andersen GE, Johnson CN, Barmuta LA, Jones ME. Use of anthropogenic linear features by two medium-sized carnivores in reserved and agricultural landscapes. Sci Rep. 2017;7:1-11.
120. Robley A, Gormley A, Forsyth DM, Wilton AN, Stephens D. Movements and habitat selection by wild dogs in eastern Victoria. Aust Mammal. 2010:32:23-32.

121. Hinton JW, Proctor C, Kelly MJ, van Manen FT, Vaughan MR, Chamberlain MJ. Space use and habitat selection by resident and transient red wolves (Canis rufus). PLoS ONE. 2016;11:e0167603.

122. Trewhella WJ, Harris S. The effect of railway lines on urban fox (Vulpes vulpes) numbers and dispersal movements. J Zool. 1990;221:321-6.

123. Svobodova J, Salek M, Albrecht T. Roads do not increase predation on experimental nests in a highly fragmented forest landscape. Folia Zool Praha. 2007;56:84.

124. Vignieri SN. Streams over mountains: influence of riparian connectivity on gene flow in the Pacific jumping mouse (Zapus trinotatus). Mol Ecol. 2005;14:1925-37.

125. Wilson A, Fenton B, Malloch G, Boag B, Hubbard S, Begg G. Urbanisation versus agriculture: a comparison of local genetic diversity and gene flow between wood mouse Apodemus sylvaticus populations in human-modified landscapes. Ecography. 2016;39:87-97.

126. Cooke RSC, Eigenbrod F, Bates AE. Projected losses of global mammal and bird ecological strategies. Nat Commun. 2019;10:1-8.

127. Ruiz-Capillas P, Mata C, Malo JE. Road verges are refuges for small mammal populations in extensively managed Mediterranean landscapes. Biol Conserv. 2013;158:223-9.

128. Rytwinski T, Fahrig L. Why are some animal populations unaffected or positively affected by roads? Oecologia. 2013;173:1143-56.

129. Ford AT, Fahrig L. Movement patterns of eastern Chipmunks (Tamias striatus) near roads. J Mammal. 2008:89:895-903.

130. McGregor RL, Bender DJ, Fahrig L. Do small mammals avoid roads because of the traffic? J Appl Ecol. 2008:45:117-23.

131. Downing RJ, Rytwinski T, Fahrig L. Positive effects of roads on small mammals: a test of the predation release hypothesis. Ecol Res. 2015;30:651-62.

132. Da Silva GR, Diniz P, Banhos A, Duca C. Positive roadside edge effects on artificial nest survival in a lowland Atlantic forest. Ecol Evol. 2019;9:7402-9.

133. Planillo A, Mata C, Manica A, Malo JE. Carnivore abundance near motorways related to prey and roadkills. J Wildl Manage. 2018;82:319-27.

134. Galantinho A, Eufrazio S, Silva C, Carvalho F, Alpizar-Jara R, Mira A. Road effects on demographic traits of small mammal populations. Eur J Wildl Res. 2017;63:22

135. Kociolek AV, Clevenger AP, Clair CCS, Proppe DS. Effects of ROAD networks on bird populations. Conserv Biol. 2011;25:241-9.

136. Arévalo JE, Newhard K. Traffic noise affects forest bird species in a protected tropical forest. Rev Biol Trop. 2011;59:969-80.

137. Reijnen R, Foppen R, Braak CT, Thissen J. The effects of car traffic on breeding bird populations in woodland. III. Reduction of density in relation to the proximity of main roads. J Appl Ecol. 1995;32:187-202.

138. Ortega CP. Effects of noise pollution on birds: a brief review of our knowledge. Ornithol Monogr. 2012;74:6-22.

139. Grade AM, Sieving KE. When the birds go unheard: highway noise disrupts information transfer between bird species. Biol Lett. 2016;12:20160113.

140. Lucass C, Eens M, Müller W. When ambient noise impairs parentoffspring communication. Environ Pollut. 2016;212:592-7.

141. Verzijden MN, Ripmeester EAP, Ohms VR, Snelderwaard P, Slabbekoorn H. Immediate spectral flexibility in singing chiffchaffs during experimental exposure to highway noise. J Exp Biol. 2010;213:2575-81.

142. Ware HE, McClure CJW, Carlisle JD, Barber JR. A phantom road experiment reveals traffic noise is an invisible source of habitat degradation. Proc Natl Acad Sci USA. 2015;112:12105-9.

143. Halfwerk W, Holleman LJM, Lessells CM, Slabbekoorn H. Negative impact of traffic noise on avian reproductive success. J Appl Ecol. 2011:48:210-9.

144. Rheindt FE. The impact of roads on birds: does song frequency play a role in determining susceptibility to noise pollution? J Ornithol. 2003:144:295-306.

145. Erritzoe J, Mazgajski TD, Rejt $Ł$. Bird casualties on European roads—a review. Acta Ornithol. 2003;38:77-93.

146. Loss SR, Will T, Marra PP. Estimation of bird-vehicle collision mortality on U.S. roads. J Wildl Manage. 2014;78:763-71. 
147. Guinard É, Julliard R, Barbraud C. Motorways and bird traffic casualties: carcasses surveys and scavenging bias. Biol Conserv. 2012;147:40-51.

148. Santos SM, Mira A, Salgueiro PA, Costa P, Medinas D, Beja P. Avian trait-mediated vulnerability to road traffic collisions. Biol Conserv. 2016;200:122-30.

149. Orlowski G. Roadside hedgerows and trees as factors increasing road mortality of birds: implications for management of roadside vegetation in rural landscapes. Landsc Urban Plan. 2008;86:153-61.

150. Sabo JL, Power ME. River-watershed exchange: effects of riverine subsidies on riparian lizards and their terrestrial prey. Ecology. 2002;83:1860-9.

151. Kato C, Iwata T, Nakano S, Kishi D. Dynamics of aquatic insect flux affects distribution of riparian web-building spiders. Oikos. 2003;103:113-20.

152. Murakami M, Nakano S. Indirect effect of aquatic insect emergence on a terrestrial insect population through by birds predation. Ecol Lett. 2002;5:333-7.

153. Chan EKW, Yu Y-T, Zhang Y, Dudgeon D. Distribution patterns of birds and insect prey in a Tropical Riparian Forest. Biotropica. 2008;40:623-9.

154. Gray LJ. Response of Insectivorous Birds to Emerging Aquatic Insects in Riparian Habitats of a Tallgrass Prairie Stream. The American Midland Naturalist. 1993;129:288-300.

155. Fukui D, Murakami M, Nakano S, Aoi T. Effect of emergent aquatic insects on bat foraging in a riparian forest. J Anim Ecol. 2006;75:1252-8.

156. Kajzer-Bonk J, Skórka P, Bonk M, Lenda M, Rożej-Pabijan E, Wantuch M, et al. The effect of railways on bird diversity in farmland. Environ Sci Pollut Res. 2019. https://doi.org/10.1007/s11356-019-06245-0.

157. Wiacek J, Polak M, Filipiuk M, Kucharczyk M, Bohatkiewicz J. Do birds avoid railroads as has been found for roads? Environ Manage. 2015;56:643-52

158. Howard SD, Bickford DP. Amphibians over the edge: silent extinction risk of data deficient species. Divers Distrib. 2014;20:837-46.

159. Cushman SA. Effects of habitat loss and fragmentation on amphibians: a review and prospectus. Biol Conserv. 2006;128:231-40.

160. Jochimsen DM, Peterson CR, Andrews KM, Gibbons JW. Literature review of the effects of roads on amphibians and reptiles. Boise: Idaho Fish and Game Department, USDA Forest Service; 2004. p. 79.

161. Encarnacao JA, Becker NI. Stealthy at the roadside: connecting role of roadside hedges and copse for silvicolous, small mammal populations. J Nat Conserv. 2015:27:37-43.

162. Friebe K, Steffens T, Schulz B, Valqui J, Reck H, Hartl G. The significance of major roads as barriers and their roadside habitats as potential corridors for hazel dormouse migration—a population genetic study. Folia Zool. 2018;67:98-109.

163. Grilo C, Del Cerro I, Centeno-Cuadros A, Ramiro V, Roman J, MolinaVacas $G$, et al. Heterogeneous road networks have no apparent effect on the genetic structure of small mammal populations. Sci Total Environ. 2016;565:706-13.

164. Mader $\mathrm{H}-\mathrm{J}$. Animal habitat isolation by roads and agricultural fields. Biol Conserv. 1984;29:81-96.

165. Rautsaw RM. The paths less traveled: movement of Gopher Tortoises (Gopherus polyphemus) along roads and railways. M.Sc thesis. University of Central Florida; 2017. https://www.researchgate.net/publicatio n/324573647_The_paths_less_traveled_movement_of_Gopher_Torto ises_Gopherus_polyphemus_along_roads_and_railways. Accessed 28 Nov 2018.

166. Latham ADM, Boutin S. Impacts of utility and other industrial linear corridors on wildlife. In: Van Der Ree R, Smith DJ, Grilo C, editors. Handbook of road ecology. Hoboken: Wiley; 2015. p. 228-36. https://doi. org/10.1002/9781118568170.ch27.

167. Gurevitch J, Curtis PS, Jones MH. Meta-analysis in ecology. Adv. Ecol Res. 2001:32:199-247.

168. Jones ME. Road upgrade, road mortality and remedial measures: impacts on a population of eastern quolls and Tasmanian devils. Wildl Res. 2000;27:289-96.

169. Azam C, Le Viol I, Julien J-F, Bas Y, Kerbiriou C. Disentangling the relative effect of light pollution, impervious surfaces and intensive agriculture on bat activity with a national-scale monitoring program. Landsc Ecol. 2016:31:2471-83.

170. Barrientos $R$, Bolonio $L$. The presence of rabbits adjacent to roads increases polecat road mortality. Biodivers Conserv. 2009;18:405-18.

171. Christie AP, Amano T, Martin PA, Shackelford GE, Simmons BI, Sutherland WJ. Simple study designs in ecology produce inaccurate estimates of biodiversity responses. J Appl Ecol. 2019;0:1-13.

172. Haddaway NR, Verhoeven JTA. Poor methodological detail precludes experimental repeatability and hampers synthesis in ecology. Ecol Evol. 2015;5:4451-4.

\section{Publisher's Note}

Springer Nature remains neutral with regard to jurisdictional claims in published maps and institutional affiliations.
Ready to submit your research? Choose BMC and benefit from:

- fast, convenient online submission

- thorough peer review by experienced researchers in your field

- rapid publication on acceptance

- support for research data, including large and complex data types

- gold Open Access which fosters wider collaboration and increased citations

- maximum visibility for your research: over 100M website views per year

At BMC, research is always in progress.

Learn more biomedcentral.com/submissions 